Nikhef 2010-019

Edinburgh 2010-021

\title{
spinney: A Form Library for Helicity Spinors
}

\author{
G. Cullen \\ The University of Edinburgh, School of Physics, Edinburgh EH9 3JZ, UK \\ M. Koch-Janusz \\ Utrecht University, 3508 TC Utrecht, The Netherlands \\ T. Reiter \\ Nikhef, Science Park 105, 1098 XG Amsterdam, The Netherlands
}

\begin{abstract}
In this work, the library spinney is presented, which provides an implementation of helicity spinors and related algorithms for the symbolical manipulation program Form. The package is well suited for symbolic amplitude calculations both in traditional, Feynman diagram based approaches and unitarity-based techniques.
\end{abstract}

Keywords: Spinor-helicity formalism, Form, Symbolical manipulation PACS: 11.80.Cr, 12.38.Bx

\section{PROGRAM SUMMARY}

Manuscript Title: spinney: A Form Library for Helicity Spinors

Authors: T. Reiter

Program Title: spinney

Email addresses: g.j.cullen@sms.ed.ac.uk (G. Cullen), maciekkj@nikhef.nl

(M. Koch-Janusz), thomasr@nikhef.nl (T. Reiter)

$U R L:$ http://www.nikhef .nl/ ${ }^{\sim}$ thomasr/ (T. Reiter) 
Programming language: Form

Keywords: Spinor-helicity formalism, Form, Symbolical manipulation

PACS: 11.80.Cr, 12.38.Bx

Classification: 4.4 Feynman diagrams, 5 Computer Algebra, 11.1 General, High Energy Physics and Computing

Nature of problem: Implementation of the spinor-helicity formalism

Solution method: Form implementation 


\section{LONG WRITE-UP}

\section{Introduction}

The success of current and future collider experiments depends on a precise prediction of the expected cross-sections for both signal processes and their corresponding backgrounds. The experimental precision of these colliders can only be matched by theoretical calculations beyond leading order in perturbation theory. Amongst the most pressing problems are the virtual (one-loop) corrections to processes with up to four particles in the final state. A disection of these one-loop amplitudes into gauge invariant subamplitudes and the use of a compact notation allows for the generation of numerically stable, fast computer programs for their evaluation. The authors of [1] introduced the spinor helicity formalism for massless particles in their cross-section calculations. Together with its extensions for massive particles, this approach leads to a compact representation of helicity amplitudes.

Although recently a number of promising purely numerical methods for the calculation of one-loop amplitudes have been presented [2 16], semi-numerical and algebraic methods are still an important tool for matrix element calculations [17-37]. The interested reader finds a more complete list of methods for one-loop ampitudes and their applications in [38, 39].

The algebraic manipulations required in matrix element calculations are very often simple, local operations that have to be applied to millions of terms, not requiring the knowledge of the whole expressions at any given moment1. General purpose computer algebra programs easily hit the memory limits when dealing with expressions of this size as they hold the whole expression in memory at every point in the program. A different approach is persued by the symbolic manipulation program Form [40]. The program provides only local manipulations of expressions, a fact which is reflected by the memory model, viewing an expression merely as a stream of terms; only a single term needs to reside in memory at any moment while the rest of the expression is stored on a storage device. In practise, a system of buffers is used to reduce the number of disk operations and multiple terms are processed at once on multi-processor systems.

\footnotetext{
${ }^{1}$ An example for a non-local algorithm is the factorization of a polynomial, which needs to know all its terms at once.
} 
The spinor helicity formalism has been implemented in the computer algebra system Mathematica through the package $S @ M$ [41]. It provides routines for the algebraic manipulation and numerical evaluation of spinor products and Dirac matrices. Mathematica's numerical and algebraic capabilities paired with a user-friendly interface are a clear advantage for many calculations. However, the above mentioned memory limitations restrict its usage to smaller problems. More involved cross-section calculations require an implementation of spinors in Form, combining the elegance of the spinor helicity formalism with Form's ability to process huge expressions. In this article we demonstrate that it is very easy to exploit Form's existing constructs

to implement helicity spinors. Therefore the implementation is in the guise of a Form library, spinney, rather than an extension of the core language. The naming of many of the functions and procedures makes reference to $S @ M$ allowing the user an easier migration between the two libraries.

This article is structured as follows: Section 2 gives an overview over the underlying theory and establishes the notation used in the rest of this work. Section 3 provides an interface documentation of the provided procedures and the defined symbols and functions. A couple of examples are discussed in Section 4 .

\section{Theoretical Background}

\subsection{Conventions}

We consider spinors $u(p)$ and $v(p)$ which are solutions of the Dirac equations

$$
(\not p-m \cdot \mathbb{I}) u(p)=0 \quad \text { and } \quad(\not p+m \cdot \mathbb{I}) v(p)=0 .
$$

Here, we denote $\mathbb{I}$ the identity operator in spinor space and $\not p=g_{\mu \nu} \gamma^{\mu} p^{\nu}$. Dimension splitting is understood in the way that for $n \in \mathbb{C}$

$$
g^{\mu \nu}=\hat{g}^{\mu \nu}+\tilde{g}^{\mu \nu}
$$

such that

$$
\begin{aligned}
\hat{g}_{\mu}^{\mu} & \equiv \hat{g}^{\mu \nu} g_{\mu \nu}=4, \\
\tilde{g}_{\mu}^{\mu} & \equiv \tilde{g}^{\mu \nu} g_{\mu \nu}=n-4 \quad \text { and } \\
\hat{g}^{\mu \rho} \tilde{g}_{\rho \nu} & =0 .
\end{aligned}
$$


The projector into the four-dimensional, physical Minkowski space is of the form

$$
\hat{g}=\operatorname{diag}(1,-1,-1,-1) \oplus \mathbf{0}^{(n-4)},
$$

i.e. it leads to the on-shell condition $\hat{p}^{2}-m^{2}=0$.

As a short-hand notation we add twiddles and hats to all vector-like objects to indicate projections into the 4 and $(n-4)$ dimensional subspaces $\left(\tilde{\gamma}^{\mu} \equiv \tilde{g}_{\nu}^{\mu} \gamma^{\nu}, \hat{k}^{\mu} \equiv \hat{g}_{\nu}^{\mu} k^{\nu}\right.$, etc. $)$. We work with anti-commuting $\gamma_{5}$ in 4 dimensions, which means

$$
\begin{aligned}
& \left\{\gamma^{\mu}, \gamma^{\nu}\right\}=2 g^{\mu \nu} \quad \text { and } \\
& \left\{\gamma_{5}, \hat{\gamma}^{\mu}\right\}=\left[\gamma_{5}, \tilde{\gamma}^{\mu}\right]=0
\end{aligned}
$$

The characteristic equation $\left(\gamma_{5}\right)^{2}-\mathbb{I}=0$ allows to introduce the usual projectors $\Pi_{L}$ and $\Pi_{R}$ into the left-handed and right-handed subspace,

$$
\Pi_{L}=\Pi_{-}=\frac{1}{2}\left(\mathbb{I}-\gamma_{5}\right) \quad \text { and } \quad \Pi_{R}=\Pi_{+}=\frac{1}{2}\left(\mathbb{I}+\gamma_{5}\right) .
$$

The helicity eigenstates of massless spinors are denoted by a commonly used bracket notation 2

$$
\begin{array}{llrl}
\Pi_{+} u\left(k_{i}\right) & =\Pi_{+} v\left(k_{i}\right)=|i\rangle, & & \left.\Pi_{-} u\left(k_{i}\right)=\Pi_{-} v\left(k_{i}\right)=\mid i\right], \\
\bar{u}\left(k_{i}\right) \Pi_{-}=\bar{v}\left(k_{i}\right) \Pi_{-}=[i \mid, & \bar{u}\left(k_{i}\right) \Pi_{+}=\bar{v}\left(k_{i}\right) \Pi_{+}=\langle i| .
\end{array}
$$

Also in the massive case we use a similar bracket notation to distinguish the different solutions of the Dirac equation. We notice that using a given lightlike vector $q$ every massive vector $p_{I}$ can be decomposed into a sum of two lightlike vectors as in the following equation

$$
p_{I}^{\mu}=k_{i}^{\mu}+\frac{\left(p_{I}\right)^{2}}{2 p_{I} \cdot q} q^{\mu}
$$

which defines the lightlike vector $k_{i}$. The solutions of the Dirac equations $\left(\not p_{I} \pm m_{I}\right)\left|I^{ \pm}\right\rangle=0$ and $\left.\left(\not p_{I} \pm m_{I}\right) \mid I^{ \pm}\right]=0$ can be expressed in terms of the

\footnotetext{
${ }^{2}$ We identify the vectors with their labels where this does not lead to ambiguities, e.g. $p_{I} \equiv I$ or $k_{j}=j$. Throughout this paper lower case Latin labels are used for light-like vectors and upper case Latin labels for massive vectors.
} 
massless spinors of $q$ and $k_{i}$,

$$
\begin{array}{ll}
\left.\left|I^{ \pm}\right\rangle=|i\rangle \pm \frac{m_{I}}{[i q]} \mid q\right], & \left.\left.\mid I^{ \pm}\right]=\mid i\right] \pm \frac{m_{I}}{\langle i q\rangle}|q\rangle, \\
\left\langle I^{ \pm}\right|=\langle i| \pm \frac{m_{I}}{[q i]}[q \mid, & {\left[I^{ \pm} \mid=\left[i \mid \pm \frac{m_{I}}{\langle q i\rangle}\langle q| .\right.\right.}
\end{array}
$$

It should be noted that in spite of the similarity in notation the massive spinors are not constructed as eigenstates of the helicity projectors $\Pi_{ \pm}$.

\subsection{Dimension Splitting}

In this section we want to derive the formulæ required for separating the dependence on $\tilde{g}$ from the spinor chains and spinor traces. The starting point for such a separation is the following equation, a proof of which can be found in [42]:

$$
\operatorname{tr}\{\mathbb{I}\} \operatorname{tr}\left\{\hat{\gamma}^{\mu_{1}} \cdots \hat{\gamma}^{\mu_{p}} \tilde{\gamma}^{\nu_{1}} \cdots \tilde{\gamma}^{\nu_{q}}\right\}=\operatorname{tr}\left\{\hat{\gamma}^{\mu_{1}} \cdots \hat{\gamma}^{\mu_{p}}\right\} \operatorname{tr}\left\{\tilde{\gamma}^{\nu_{1}} \cdots \tilde{\gamma}^{\nu_{q}}\right\}
$$

A chain of Dirac matrices can always be sorted by splitting $\gamma^{\mu}=\hat{\gamma}^{\mu}+\tilde{\gamma}^{\mu}$ and shuffling $\hat{\gamma}$ and $\tilde{\gamma}$ to opposite ends of the chain using Equation (4). The presence of $\gamma_{5}$ does not change the above results as in our scheme it can be written as $\gamma_{5}=i \epsilon_{\mu \nu \rho \sigma} \hat{\gamma}^{\mu} \hat{\gamma}^{\nu} \hat{\gamma}^{\rho} \hat{\gamma}^{\sigma} / 4$ !.

Equation (9) extends to the case of spinor chains by the observation that for any pair of massless spinors delimiting a product of Dirac matrices one can introduce light-like auxiliary vectors $p$ and $q$ in order to turn the spinor chain into a trace.

$$
\begin{aligned}
& \langle i|\cdots| j]=\frac{\operatorname{tr}\left\{\Pi_{-} \not k_{i} \cdots \not k_{j} \not p\right\}}{\langle j|p| i]},\langle i|\cdots| j\rangle=\frac{\operatorname{tr}\left\{\Pi_{-} \not k_{i} \cdots \not k_{j} \not p \not q\right\}}{[j|\not p q| i]} \\
& {[i|\cdots| j\rangle=\frac{\operatorname{tr}\left\{\Pi_{+} \not \not_{i} \cdots \not k_{j} \not p\right\}}{[j|\not| i\rangle}, \quad[i|\cdots| j]=\frac{\operatorname{tr}\left\{\Pi_{+} \not k_{i} \cdots \not k_{j} \not p q\right\}}{\langle j|\not p q| i\rangle}}
\end{aligned}
$$

In all four cases, the insertion can be undone after Equation (9) has been applied and hence no spurious denominators need to be inserted in an actual calculation.

The trace of $(n-4)$ dimensional Dirac matrices $\tilde{\gamma}$ can be evaluated according to the recursion relation

$$
\operatorname{tr}\left\{\tilde{\gamma}^{\nu_{1}} \ldots \tilde{\gamma}^{\nu_{q}}\right\}=\sum_{i}=2^{q}(-1)^{i} \tilde{g}^{\nu_{1} \nu_{i}} \operatorname{tr}\left\{\tilde{\gamma}^{\nu_{2}} \ldots \tilde{\gamma}^{\nu_{i-1}} \tilde{\gamma}^{\nu_{i+1}} \ldots \tilde{\gamma}^{\nu_{q}}\right\}
$$


Since only even numbers of $\tilde{\gamma}$ lead to non-vanishing contributions, a chain with two Dirac matrices can be expanded according to the above rules into

$$
\left\langle i\left|\gamma^{\mu} \gamma^{\nu}\right| j\right\rangle=\left\langle i\left|\hat{\gamma}^{\mu} \hat{\gamma}^{\nu}\right| j\right\rangle+\left\langle i\left|\tilde{\gamma}^{\mu} \tilde{\gamma}^{\nu}\right| j\right\rangle=\left\langle i\left|\hat{\gamma}^{\mu} \hat{\gamma}^{\nu}\right| j\right\rangle+\tilde{g}^{\mu \nu}\langle i j\rangle
$$

As a more complicated example, we also give an explicit expression for a spinor chain with four matrices:

$$
\begin{array}{r}
{\left[i\left|\gamma^{\mu} \gamma^{\nu} \gamma^{\rho} \gamma^{\sigma}\right| j\right]=\left[i\left|\hat{\gamma}^{\mu} \hat{\gamma}^{\nu} \hat{\gamma}^{\rho} \hat{\gamma}^{\sigma}\right| j\right]+\tilde{g}^{\rho \sigma}\left[i\left|\hat{\gamma}^{\mu} \hat{\gamma}^{\nu}\right| j\right]-\tilde{g}^{\nu \sigma}\left[i\left|\hat{\gamma}^{\mu} \hat{\gamma}^{\rho}\right| j\right]} \\
+\tilde{g}^{\nu \rho}\left[i\left|\hat{\gamma}^{\mu} \hat{\gamma}^{\sigma}\right| j\right]+\tilde{g}^{\mu \sigma}\left[i\left|\hat{\gamma}^{\nu} \hat{\gamma}^{\rho}\right| j\right]-\tilde{g}^{\mu \rho}\left[i\left|\hat{\gamma}^{\nu} \hat{\gamma}^{\sigma}\right| j\right]+\tilde{g}^{\mu \nu}\left[i\left|\hat{\gamma}^{\rho} \hat{\gamma}^{\sigma}\right| j\right] \\
+[i j]\left(\tilde{g}^{\mu \nu} \tilde{g}^{\rho \sigma}-\tilde{g}^{\mu \rho} \tilde{g}^{\nu \sigma}+\tilde{g}^{\mu \sigma} \tilde{g}^{\nu \rho}\right)
\end{array}
$$

\subsection{Chisholm Identities}

With the same argument as in the previous section, we can extend the validity of the Chisholm identity

$$
\operatorname{tr}\left\{\hat{\gamma}^{\mu_{1}} \cdots \hat{\gamma}^{\mu_{2 m-1}} \hat{\gamma}^{\nu}\right\} \hat{\gamma}_{\nu}=2\left(\hat{\gamma}^{\mu_{1}} \cdots \hat{\gamma}^{\mu_{2 m-1}}+\hat{\gamma}^{\mu_{2 m-1}} \cdots \hat{\gamma}^{\mu_{1}}\right)
$$

to the case of spinor chains by substituting Equation (10). If we denote products of Dirac matrices as $\Gamma=\hat{\gamma}^{\mu_{1}} \cdots \hat{\gamma}^{\mu_{p}}$ and $\Gamma^{\prime}=\hat{\gamma}^{\nu_{1}} \cdots \hat{\gamma}^{\nu_{q}}$ and their reversed strings by $\overleftarrow{\Gamma}=\hat{\gamma}^{\mu_{p}} \cdots \hat{\gamma}^{\mu_{1}}$, the Chisholm identities for spinor chains read

$$
\begin{aligned}
& \left\langle i\left|\Gamma \hat{\gamma}^{\mu} \Gamma^{\prime}\right| j\right\rangle \cdot \hat{\gamma}_{\mu}=2 \Gamma^{\prime}|j\rangle\langle i|\Gamma-2 \overleftarrow{\Gamma}| i\rangle\langle j| \overleftarrow{\Gamma^{\prime}} \\
& \left.\left[i\left|\Gamma \hat{\gamma}^{\mu} \Gamma^{\prime}\right| j\right] \cdot \hat{\gamma}_{\mu}=2 \Gamma^{\prime} \mid j\right][i|\Gamma-2 \overleftarrow{\Gamma}| i]\left[j \mid \overleftarrow{\Gamma^{\prime}}\right. \\
& \left.\left\langle i\left|\Gamma \hat{\gamma}^{\mu} \Gamma^{\prime}\right| j\right] \cdot \hat{\gamma}_{\mu}=2 \Gamma^{\prime} \mid j\right]\langle i|\Gamma+2 \overleftarrow{\Gamma}| i\rangle\left[j \mid \overleftarrow{\Gamma^{\prime}}\right. \\
& {\left[i\left|\Gamma \hat{\gamma}^{\mu} \Gamma^{\prime}\right| j\right\rangle \cdot \hat{\gamma}_{\mu}=2 \Gamma^{\prime}|j\rangle[i|\Gamma+2 \overleftarrow{\Gamma}| i]\langle j| \overleftarrow{\Gamma^{\prime}}}
\end{aligned}
$$

The above identities are valid only if the number of Dirac matrices in $\Gamma$ and $\Gamma^{\prime}$ matches the helicities of the spinors $i$ and $j$, i.e. in equations (15a) and (15b) the length of $\Gamma \hat{\gamma}^{\mu} \Gamma^{\prime}$ must be even 3 and in (15c) and (15d) it must be odd.

The repeated application of Equation (15) together with the identities

$$
\hat{\gamma}^{\mu} \Gamma \hat{\gamma}_{\mu}=-2 \overleftarrow{\Gamma} \quad \text { and } \quad \hat{\gamma}^{\mu} \Gamma \hat{\gamma}^{\nu} \hat{\gamma}_{\mu}=2\left(\hat{\gamma}^{\nu} \Gamma+\overleftarrow{\Gamma} \hat{\gamma}^{\nu}\right)
$$

\footnotetext{
${ }^{3} \gamma_{5}$ counts as an even number of matrices
} 
for an odd number of matrices in $\Gamma$, ensures that, starting from an expression with no uncontracted Lorentz indices, all spinor chains in four dimensions and traces can be expressed in terms of spinor products of the form $\langle i j\rangle$ and $[i j]$.

\subsection{Majorana Spinors}

In supersymmetric extensions of the standard model we need to deal with interactions of Majorana fermions. A Majorana fermion is its own antiparticle, i.e. it is invariant under charge conjugation:

$$
\widetilde{\psi}_{M}=C \bar{\psi}_{M}^{T}=\psi_{M}
$$

The charge-conjugation matrix has the properties:

$$
C^{\dagger}=C^{-1}, \quad C^{T}=-C, \quad C \Gamma_{i}^{T} C^{-1}=\eta_{i} \Gamma_{i}
$$

with

$$
\eta_{i}=\left\{\begin{array}{l}
+1 \text { for } \Gamma_{i}=1, \gamma_{5}, \gamma_{\mu} \gamma_{5} \\
-1 \text { for } \Gamma_{i}=\gamma_{\mu}
\end{array}\right.
$$

We aim to write down a consistent set of Feynman rules to deal with Majorana fermions. The problem is that vertices involving Majorana fermions violate fermion number flow; the fermion flow in the Feynman diagram is illdefined. A consistent way of dealing with this was proposed in [43]. There are two drawbacks with this approach; charge conjugation matrices are explicitly introduced into the Feynman rules and the relative sign of the Feynman graphs needs to be determined from the original Wick contractions.

We follow the approach in [44] which has been implemented in [45]. Each vertex containing Dirac fermions has two expressions, one in which the fermion flow follows the fermion number flow, and the other "flipped" vertex, where the fermion flows in the opposite direction to the fermion number flow. In our implementation we impose a fermion flow on the vertex through the procedure RemoveNCContainer and "flip" the vertices and spinors where neccesary.

We have implemented the following flipping rules, using (19):

$$
\begin{gathered}
\left(\gamma^{\mu}\right)^{\prime}=-\gamma^{\mu} \\
\left(\Pi_{ \pm}\right)^{\prime}=\Pi_{ \pm}
\end{gathered}
$$


and for the spinors

$$
\begin{aligned}
& |p\rangle^{\prime}=\langle p| \\
& \mid p]^{\prime}=[p \mid .
\end{aligned}
$$

This leads to the following rule for the fermionic propagator:

$$
S^{\prime}(p)=\frac{1}{-\not p-m}=S(-p) .
$$

Another appealing feature of this method is that the relative sign between graphs can be determined directly from the expressions as opposed to reverting back to the original Wick contractions. This brings it in line with the usual formulation for Dirac fermions. To compute a consistent relative sign of Feynman graphs each must be multiplied by $(-1)^{P+L}$, with,

- P: the parity of the permutation of external spinors with respect to some reference order,

- L: the number of closed fermion loops.

This sign is determined after the flipping rules are applied.

\section{Program Description}

\subsection{Installation}

The library has been written using the literate programming tool nuweb [46]. It can be obtained by downloading the file spinney.tgz from the URL http://www.nikhef.nl/ thomasr/filetransfer.php=spinney.tgz . The tarball contains the following files:

spinney.hh the Form file

spinney.pdf annotated source code

spinney.nw the nuweb sources

spinney_test.frm unit tests

After unpacking the library the should run the test program spinney_test.frm in order to ensure that the installed Form version is recent enough. If one or more tests in the program fail the most likely reason is a deprecated version of Form.

The file spinney.nw is only needed if the user wants to rebuild any of the other files from scratch. 


\subsection{Representation in Form}

The spinney library uses four different representations for spinorial objects, each of which is considered the most convenient form at a given point in the workflow.

For the description of the definitions and declarations we use the Form keywords (CFunction, NFunction, CTensor, NTensor, Vector, Symbol, \#Procedure) in the text to indicate its type. Where more than one definition is combined in one box the arguments apply to all of the definitions.

\subsubsection{Non-Commuting Objects}

The 't Hooft algebra and the dimension splitting are applied to Form's non-commuting object. In order to keep the information about the dimension in the function names rather than the indices we do not use Form's Dirac matrices here (g-) but introduce our own functions.

\begin{tabular}{|c|}
\hline NTensor $\operatorname{Sm}(\mu)$ \\
\hline An $n$-dimensional Dirac matrix $\gamma^{\mu}$. \\
\hline Parameters \\
\hline$\mu:$ Lorentz index or vector \\
\hline NTensor $\operatorname{Sm} 4, \operatorname{SmEps}(\mu)$ \\
\hline $\begin{array}{l}\text { The 4-dimensional and }(n-4) \text {-dimensional (resp.) projections } \\
\hat{\gamma}^{\mu} \text { and } \tilde{\gamma}^{\mu} \text { of the Dirac matrix } \gamma^{\mu} \text {. }\end{array}$ \\
\hline
\end{tabular}

The non-commuting objects corresponding to the four-dimensional matrix $\gamma_{5}$ and the derived projectors $\Pi_{ \pm}=\left(\mathbb{I} \pm \gamma_{5}\right) / 2$ are described below; they have been implemented as non-commuting functions without parameters.

\begin{tabular}{l}
\hline NFunction Gamma5() \\
The Dirac matrix $\gamma_{5}$. \\
\begin{tabular}{l} 
NFunction ProjPlus, ProjMinus () \\
\hline The projectors $\Pi_{R}=\Pi_{+}=\left(\mathbb{I}+\gamma_{5}\right) / 2$ and \\
$\Pi_{L}=\Pi_{-}=\left(\mathbb{I}-\gamma_{5}\right) / 2$ (resp.)
\end{tabular}
\end{tabular}

In the case of the spinors we use the same function names for both massive and massless spinors. Massive spinors are distinguished by their second 
argument $\rho$, which is \pm 1 , indicating that the spinor lies in the kernel of the operator $(\not p-\rho m)$. For massless spinors the second argument must be omitted.

NFunction USpa, $\operatorname{UbarSpb}(p[, \rho])$

The massless spinor $|p\rangle$ and its conjugated $[p \mid$, if $\rho$ is omitted; the massive spinor $\left|p^{\rho}\right\rangle$ and its conjugated $\left[p^{\rho} \mid\right.$ otherwise.

\section{Parameters}

$p$ : momentum of the spinor

$\rho$ : (optional), a value \pm 1 as described above

NFunction USpb, $\operatorname{UbarSpa}(p[, \rho])$

The massless spinor $[p]$ and its conjugated $\langle p|$, if $\rho$ is omitted; the massive spinor $\left.\mid p^{\rho}\right]$ and its conjugated $\left\langle p^{\rho}\right|$ otherwise.

\section{Parameters}
$p$ : momentum of the spinor
$\rho$ : (optional), a value \pm 1 as described above

The following two functions mark the beginning and the end of a Dirac trace.

\begin{tabular}{|l|}
\hline NFunction $\operatorname{trL}, \operatorname{trR}()$ \\
\hline Indicates the begin (resp. end) of a Dirac trace. \\
\hline
\end{tabular}

For example, the expression $\operatorname{tr}\left\{\Pi_{+} \gamma^{\mu} \not \gamma^{\nu} \not\right\}$ would correspond to the following product in Form:

$\operatorname{trL} * \operatorname{ProjPlus} * \mathrm{Sm}(\mathrm{mu}) * \mathrm{Sm}(\mathrm{p}) * \mathrm{Sm}(\mathrm{nu}) * \mathrm{Sm}(\mathrm{q}) * \operatorname{trR}$

\subsubsection{Indexed Notation}

In this form the spinor indices of Dirac matrices and spinors are kept. For example, the term $\left\langle k_{1}|\not| k_{2}\right]$ would be expressed as

$$
\left.\left(\left\langle k_{1}\right|\right)_{\alpha_{1}}(\not p)_{\alpha_{1} \alpha_{2}}\left(\mid k_{2}\right]\right)_{\alpha_{2}}
$$

and in a Form program as 


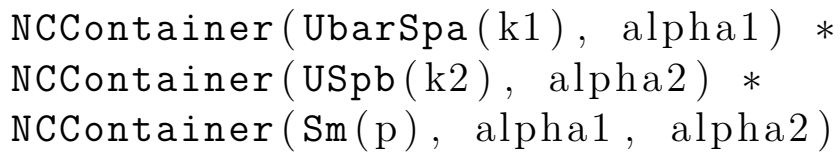

This notation is particularly useful when importing expressions from a diagram generator, which does not necessarily put the factors in the correct order. All elements of the spinor line are wrapped inside the function NCContainer which is defined as follows.

CFunction NCContainer $\left(o, i_{1}\left[, i_{2}\right]\right)$

Representation of a spinor or a Dirac matrix with explicit spinor indices.

\section{Parameters}

$o$ : a non-commuting object, such as Sm, Gamma5 or UbarSpa; a complete list is given in Section 3.2.1.

$i_{1}$ : for spinors: the only spinor index; for Dirac matrices: the first spinor index

$i_{2}$ : for spinors: not present; for Dirac matrices: the second spinor index

\subsubsection{Collected Form}

The collected form is generated from non-commutative objects by the procedure SpCollect or from the open form by the procedure SpClose. Spinor strings are represented by the following four functions

CFunction Spaa, Spab, Spba, $\operatorname{Spbb}\left(k_{1}, \ldots, k_{2}\right)$

A string delimited by two spinors. The four forms correspond to $\left\langle k_{1}|\ldots| k_{2}\right\rangle,\left\langle k_{1}|\ldots| k_{2}\right],\left[k_{1}|\ldots| k_{2}\right\rangle$ and $\left[k_{1}|\ldots| k_{2}\right]$ respectively.

\section{Parameters}

$k_{1}$ : a light-like, four-dimensional vector.

...: a list of four-dimensional indices or vectors.

$k_{2}$ : a light-like, four-dimensional vector. 


\subsubsection{Open Form}

The open form can be generated from the collected form by calling the procedure SpOpen after all Lorentz indices have been contracted using SpContract. The reverse operation of SpOpen is SpClose, which transforms the open form back to collected form.

CFunction $\operatorname{Spa} 2, \operatorname{Spb} 2\left(k_{1}, k_{2}\right)$

The spinor products $\left\langle k_{1} k_{2}\right\rangle$ and $\left[k_{1} k_{2}\right]$ respectively. These functions are defined as anti-symmetric in their arguments.

\section{Parameters}

$k_{1}$ : a light-like, four-dimensional vector.

$k_{2}$ : a light-like, four-dimensional vector.

\subsubsection{Metric Tensors}

The dimension splitting of $g^{\mu \nu}$ into the sum $\hat{g}^{\mu \nu}+\tilde{g}^{\mu \nu}$ as described in Equation (2) is reflected in the definition of the metric tensor and its projections onto subspaces. As in the case of the Dirac matrices we find it more convenient to define new functions for the metric tensor rather than using the tensor $\mathrm{d}_{-}$, which is predefined in Form.

CTensor $\mathrm{d}(\mu, \nu)$

The $n$-dimensional metric tensor $g^{\mu \nu}$. This function is defined symmetric in its arguments.

CTensor d4, dEps $(\mu, \nu)$

$\mathrm{d} 4$ is the 4-dimensional projection $\hat{g}^{\mu \nu}$ of the metric tensor; $\mathrm{dEps}$ is the orthogonal projection $\tilde{g}^{\mu \nu}$ into the $(n-4)$ dimensional subspace.

These function are defined symmetric in their arguments.

\subsubsection{Other Symbols and Objects}

The function SpDenominator has been introduced in order to represent the reciprocal value of its argument. Therefore, the replacement SpDenominator $(x$ ? $) \rightarrow$ $1 / x$ is always safe. However, keeping certain denominators inside function arguments makes some substitutions easier. 
CFunction SpDenominator $(x)$

Represents the reciprocal value of the argument, i.e. $1 / x$.

The function SpERRORTOKEN has been introduced to indicate inconsitencies detected by the procedure SpCheck which signals if the length of a spinor string trivially nullifies an expression. Although these null-expressions are not errors, in certain circumstances they can indicate errors in a program.

\section{CFunction SpERRORTOKEN()}

Indicates that the procedure SpCheck found an error in the term where SPERRORTOKEN appears.

\subsection{Reserved Symbols}

The library spinney defines more objects for internal use. All implemented algorithms require that none of these objects are present in any active expression at the invocation of the procedure and ensure that these objects are not present in any active expression after the procedure returns. These objects are declared exactly as written below.

CFunctions fDUMMY1, ..., fDUMMY4;

Symbols sDUMMY1, .., sDUMMY4;

Indices iDUMMY1, ..., iDUMMY4;

Vectors vDUMMY1, ..., vDUMMY4;

NFunctions nDUMMY1, SpFlip;

We also define the three sets SpORIGSet, SpIMAGSet and SpObject which are used inside RemovenCContainer.

\subsection{Implemented Algorithms}

\subsubsection{Light-Cone Decomposition}

This algorithm uses Equations (77) and (8) in order to express massive vectors and spinors in terms of massless ones. 
\#Procedure LightConeDecomposition $\left(p_{I}, p_{i}, q, m_{I}\right)$

In every active expression all appearances of $p_{I}$ and all spinors $\left.\left|I^{ \pm}\right\rangle, \mid I^{ \pm}\right],\left\langle I^{ \pm}\right|$and $\left[I^{ \pm} \mid\right.$are replaced by a pair of light-like vectors (resp. spinors).

The parameters are defined according to Equation (7):

\section{Parameters}

$p_{I}: \quad$ a massive vector that fulfills $\left(p_{I}\right)^{2}=m_{I}^{2}$

$p_{i}$ : a light-like vector

$q: \quad$ a light-like vector (reference momentum)

$m_{I}:$ the mass of $p_{I}$

This procedure works on non-commuting objects. If massive spinors are present this procedure must be called before SpCollect.

This procedure needs to be called before SpCollect as in the closed form only massless spinors are allowed.

As an example, we write down the numerator of the color stripped tree level diagram of $u \bar{u} \rightarrow t \bar{t}$ in QCD for two different helicities.

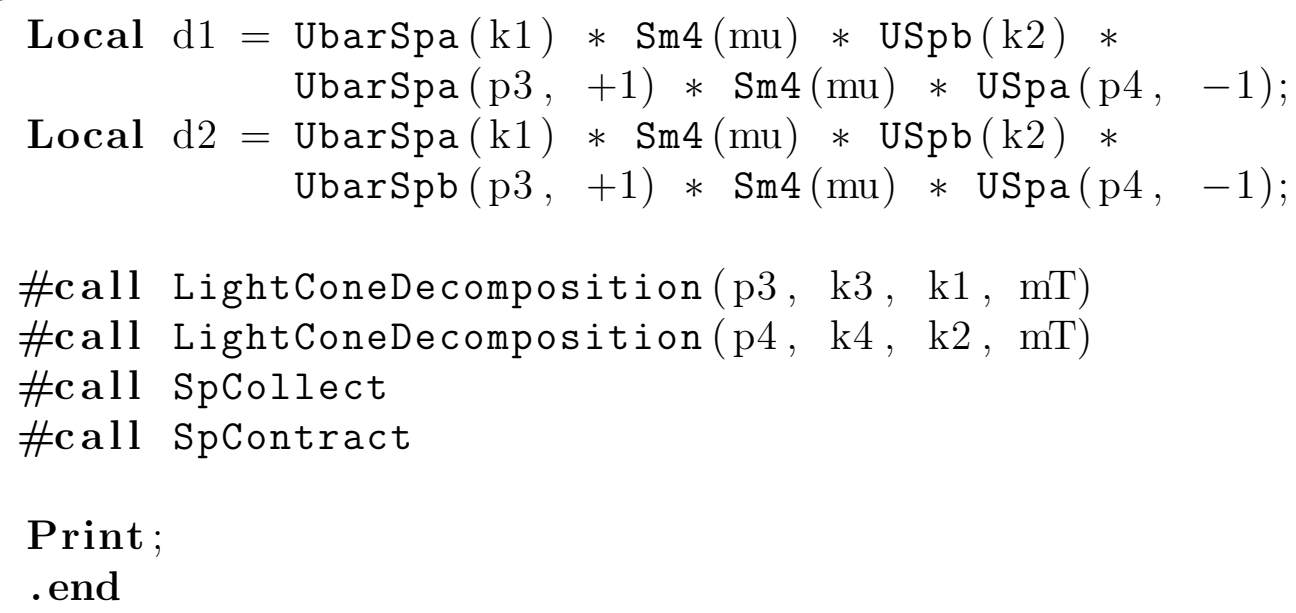

We have also added the command SpContract in this example as it simplifies the output considerably, which is given below. 


$$
\begin{aligned}
& \mathrm{d} 1= 2 * \operatorname{Spaa}(\mathrm{k} 1, \mathrm{k} 4) * \operatorname{Spbb}(\mathrm{k} 1, \mathrm{k} 2) * \\
& \operatorname{SpDenominator}(\operatorname{Spb} 2(\mathrm{k} 1, \mathrm{k} 3)) * \mathrm{mT} \\
& \mathrm{d} 2= 2 * \operatorname{Spa}(\mathrm{k} 1, \mathrm{k} 4) * \operatorname{Spbb}(\mathrm{k} 3, \mathrm{k} 2) \\
& 0.00 \text { sec out of } 0.03 \mathrm{sec}
\end{aligned}
$$

The above calculation can therefore be summarized as:

$$
\begin{aligned}
& d_{1}=\left\langle k_{1}\left|\hat{\gamma}^{\mu}\right| k_{2}\right]\left\langle p_{3}^{+}\left|\hat{\gamma}_{\mu}\right| p_{4}^{-}\right\rangle=2 \cdot \frac{\left\langle k_{1} k_{4}\right\rangle\left[k_{1} k_{2}\right]}{\left[k_{1} k_{3}\right]} \cdot m_{T} \\
& d_{2}=\left\langle k_{1}\left|\hat{\gamma}^{\mu}\right| k_{2}\right]\left\langle p_{3}^{+}\left|\hat{\gamma}_{\mu}\right| p_{4}^{-}\right]=2 \cdot\left\langle k_{1} k_{4}\right\rangle\left[k_{3} k_{2}\right]
\end{aligned}
$$

\subsection{2. n-Dimensional 't Hooft Algebra}

\#Procedure tHooftAlgebra()

Carries out the $(n-4)$-dimensional part of the algebra such that only four dimensional Dirac matrices $\hat{\gamma}^{\mu}$ are left; the dependence on $(n-4)$ is entirely expressed in terms of $\tilde{g}^{\mu \nu}$.

This procedure works on non-commuting objects. If

$n$-dimensional Dirac matrices, $\Pi_{ \pm}$or $\gamma_{5}$ are present this procedure must be called before SpCollect.

As an example, we give a short program that reproduces Equations (12) and (13).

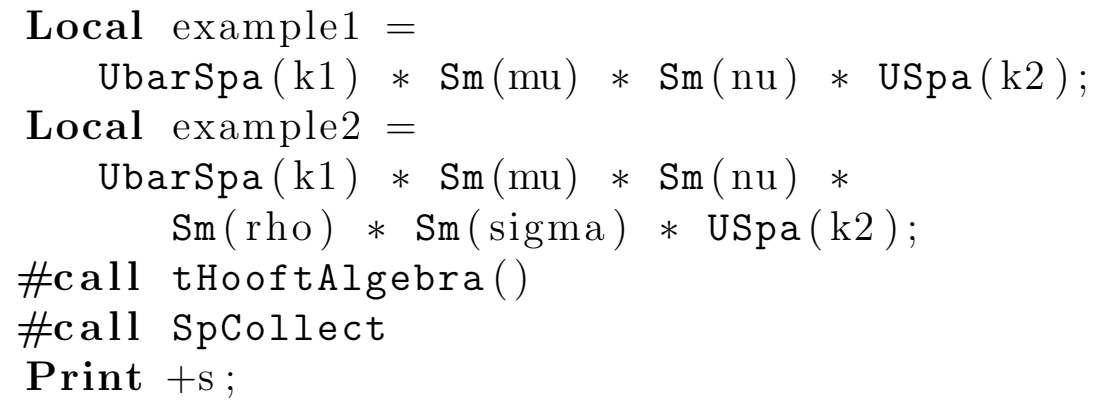

\subsubsection{Change of Representation}

As described in Section 3.2, different representations for the expressions are used at different points in a Form program using spinney. Here, we give an overview of the functions changing between the representations. While for most representations only one-directional translation is provided, the user 
can change forth and back between collected form and open form, which is indicated in the diagram below

$$
\begin{gathered}
\text { NCContainer }(\operatorname{UbarSpa}(\mathrm{p}), \mathrm{i}) * \operatorname{NCContainer}(\operatorname{USpa}(\mathrm{q}), \mathrm{i}) \stackrel{\text { RemovenCContainer }}{\longrightarrow} \stackrel{\mathrm{U}}{\longrightarrow} \mathrm{UbarSpa}(\mathrm{p}) * \mathrm{USpa}(\mathrm{b}) \stackrel{\text { SpCollect }}{\longrightarrow} \operatorname{Spaa}(\mathrm{p}, \mathrm{q}) \underset{\mathrm{SpClose}}{\stackrel{\text { SpOpen }}{\longrightarrow}} \operatorname{Spa} 2(\mathrm{p}, \mathrm{q})
\end{gathered}
$$

The first in this collection of procedures is RemoveNCContainer. Its effect amounts to stripping off the function NCContainer and ordering the Dirac matrices and spinors according to the order of the spinor indices. Where necessary the flipping rules for Majorana fermions are applied (see Section 2.4).

$$
\text { \#Procedure RemoveNCContainer() }
$$

Translates the indexed notation into non-commuting objects.

At the level of non-commuting objects the dimension splitting and the 't Hooft algebra take place, which has been implemented in the routine tHooftAlgebra.

After these steps, typically, one wants to proceed in collected form. This form is better suited for contractions across different spinor lines, as in $\left\langle p_{1}\left|\hat{\gamma}^{\mu}\right| p_{2}\right]\left\langle p_{3}\left|\hat{\gamma}_{\mu}\right| p_{4}\right]$, which can be detected very easily using argument lists but is very difficult to be carried out using products of non-commuting objects.

Traces of the form trL $\cdots \operatorname{trR}$ can be converted to collected form using the procedure SpTrace4, all remaining products of non-commuting objects can be transformed by the procedure SpCollect.

\#Procedure $\operatorname{SpTrace} 4\left(k_{1}, k_{2}, \ldots\right)$

Operates on non-commutative objects in products representing a trace $(\operatorname{trL} * \ldots * \operatorname{trR})$.

\section{Parameters}

$k_{1}, k_{2}, \ldots:$ an optional list of vectors. If specified, these vectors are assumed lightlike and traces are opened at those positions. If no such positions are found the trace is evaluated using Form's Trace4 command. 
The following example demonstrates the difference in treatment between light-like vectors and those which have not been specified as light-like.

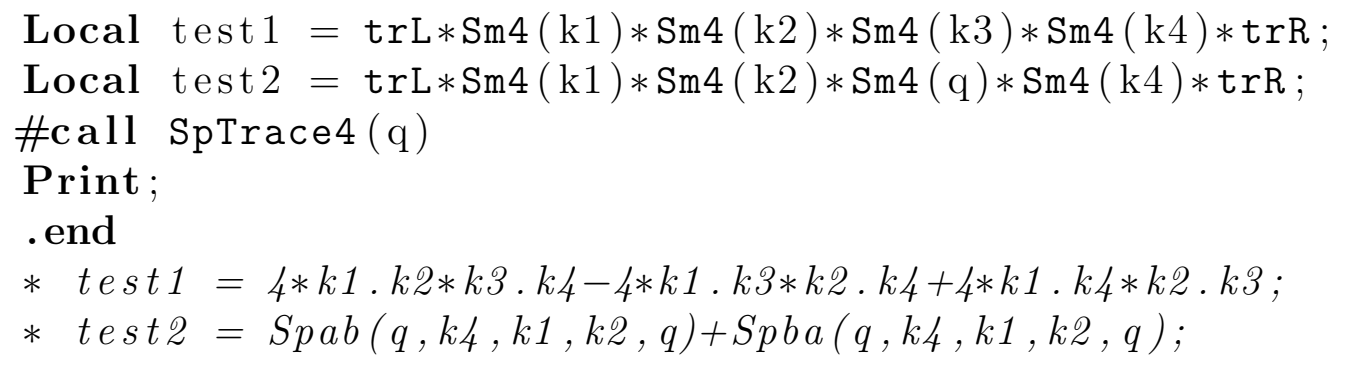

Very often specifying the list of light-like vectors leads to more compact expressions, especially if the number of terms generated by taking the trace is very big.

\#Procedure SpCollect()

Translates non-commuting objects into collected form.

This procedure requires that the $n$-dimensional algebra has already been carried out by a call to tHooftAlgebra and that all massive spinors have been replaced using the routine LightConeDecomposition.

As a result the all spinorial objects are expressed in terms of the function Spaa, Spab, Spba and Spab.

Before one can go from collected form to open form one should eliminate all Lorentz contractions by the use of SpContract and SpContractLeviCivita. The latter one is only needed if the Levi-Civita symbol $\epsilon^{\mu \nu \rho \sigma}$ has been introduced by taking a trace involving $\gamma_{5}$.

The conversion from collected to open form is performed by the procedure Sp0pen. The reverse operation, converting from open form back to collected form is done by the procedure SpClose. 
\#Procedure $\operatorname{SpOpen}\left(k_{1}, k_{2}, \ldots\right)$

Translates an expression in collected form into open form.

In open form, only the functions $\mathrm{Spa} 2$ and $\mathrm{Spb} 2$ are used to express spinor products.

This procedure requires that all Lorentz indices inside spinor lines have been removed using the routine SpContract.

\section{Parameters}

$$
\begin{aligned}
& k_{1}, k_{2}, \ldots \text { : an optional list of vectors. If } \\
& \text { specified, the spinor lines are only opened } \\
& \text { at positions indicated by the given vectors. } \\
& \text { In this case a complete translation into } \\
& \text { Spa2 and Spb2 functions might not }
\end{aligned}
$$

The parameter list of SpClose allows to close spinor strings at the positions of certain vectors, indicated by the arguments. In both cases, SpOpen and $\mathrm{SpClose}$, the parameter lists facilitate the use of this library for unitarity based methods, as will be shown in the examples of Section 4.3.

$$
\begin{aligned}
& \text { \#Procedure } \operatorname{SpClose}\left(k_{1}, k_{2}, \ldots\right) \\
& \text { The inverse operation of Sp0pen. Replaces the functions Spa2 } \\
& \text { and Spb2 into the functions Spaa, Spab etc. } \\
& \text { Parameters } \\
& \qquad k_{1}, k_{2}, \ldots \text { : an optional list of vectors. If } \\
& \text { specified, only those positions are closed } \\
& \text { which are indicated by the given vectors. }
\end{aligned}
$$

In some cases this operation requires that a spinor string is broken up at another position. Consider the following example:

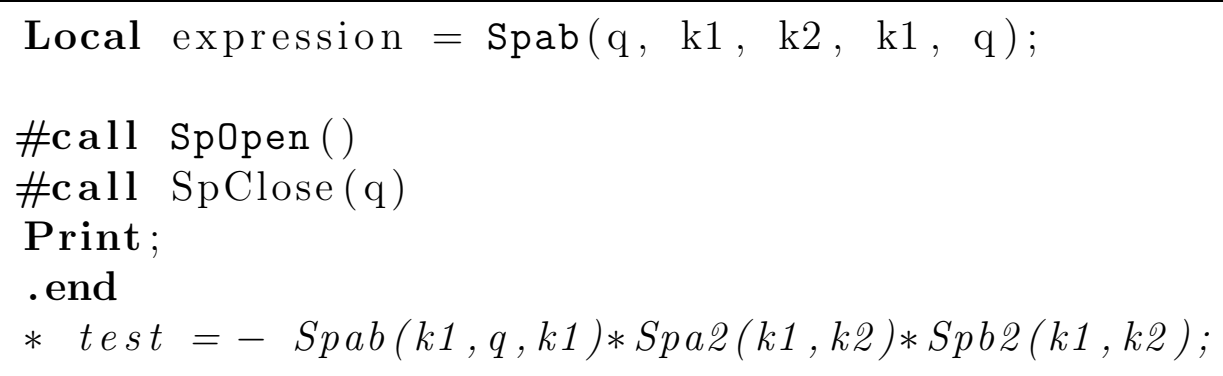


However, it is not guaranteed that the operation always succeeds: if we had written \# call SpClose(q,k1) instead of \#call SpClose(q) the following result would have come out, where the vector q still appears in the spinors.

* test $=\operatorname{Spab}(k 2, k 1, k 2) * \operatorname{Spab}(q, k 1, q)$;

\subsubsection{Contraction of Lorentz Indices}

This Section describes three routines which reduce the number of explicit appearances of contracted Lorentz indices. The first type of contractions, those involving only spinor lines, as in $\left\langle p_{1}\left|\cdots \hat{\gamma}^{\mu} \cdots\right| p_{2}\right]\left\langle p_{3}\left|\cdots \hat{\gamma}_{\mu} \cdots\right| p_{4}\right]$ or in $\left\langle p_{1}\left|\cdots \hat{\gamma}^{\mu} \cdots \hat{\gamma}_{\mu} \cdots\right| p_{2}\right]$, are treated by the procedure SpContract.

\#Procedure SpContract()

Applies the equations of Section 2.3 in order to eliminate all Lorentz indices inside spinor lines. This procedure works on expressions in collected form.

Contractions involving metric tensors are simplified by the procedure SpContractMetrics. This procedure is implemented such that it can deal with expressions in collected form but also with non-commuting objects.

\#Procedure SpContractMetrics()

Removes spurious appearances of the metric tensors $g^{\mu \nu}, \hat{g}^{\mu \nu}$ and $\tilde{g}^{\mu \nu}$. Whereas $\hat{g}_{\mu}^{\mu}=4$ is substituted immediately, all other instances of the dimensions $\tilde{g}_{\mu}^{\mu}$ and $g_{\mu}^{\mu}$ are not replaced.

The last one in this category of procedures is SpContractLeviCivita. This routine uses the fact that the Levi-Civita tensor $\epsilon^{\mu \nu \rho \sigma}$ can be written as a trace:

$$
\epsilon^{\mu \nu \rho \sigma}=-\frac{i}{4} \operatorname{tr}\left\{\gamma_{5} \hat{\gamma}^{\mu} \hat{\gamma}^{\nu} \hat{\gamma}^{\rho} \hat{\gamma}^{\sigma}\right\}
$$

The procedure considers two cases. If the Levi-Civita tensor is contracted with a Dirac matrix inside a spinor string it uses the Chisholm identity to simplify

$$
\epsilon^{\mu \nu \rho \sigma} \hat{\gamma}_{\sigma}=-\frac{i}{2}\left(\Pi_{+}-\Pi_{-}\right)\left[\hat{\gamma}^{\mu} \hat{\gamma}^{\nu} \hat{\gamma}^{\rho}-\hat{\gamma}^{\mu} \hat{\gamma}^{\nu} \hat{\gamma}^{\rho}\right]
$$

The second case is the contraction of the Levi-Civita tensor with a lightlike momentum. Here one can rewrite the $\epsilon$-tensor as

$$
p_{\mu} \epsilon^{\mu \nu \rho \sigma}=-\frac{i}{4}\left(\left[p\left|\hat{\gamma}^{\nu} \hat{\gamma}^{\rho} \hat{\gamma}^{\sigma}\right| p\right\rangle-\left\langle p\left|\hat{\gamma}^{\nu} \hat{\gamma}^{\rho} \hat{\gamma}^{\sigma}\right| p\right]\right)
$$


Products of multiple $\epsilon$-tensors are reduced by applying the determinant relation

$$
\epsilon^{\mu_{1} \mu_{2} \mu_{3} \mu_{4}} \epsilon^{\nu_{1} \nu_{2} \nu_{3} \nu_{4}}=\operatorname{det}\left(\hat{g}^{\mu_{i} \nu_{j}}\right)_{i, j=1}^{4}
$$

\#Procedure SpContractLeviCivita $\left(k_{1}, k_{2}, \ldots\right)$

Eliminates Levi-Civita tensors as far as possible applying Equations (25), (26) and (27).

\section{Parameters}

$$
\begin{aligned}
& k_{1}, k_{2}, \ldots: \text { an optional list of vectors. If } \\
& \text { specified, these vectors are assumed } \\
& \text { lightlike. }
\end{aligned}
$$

\subsubsection{Schouten Identity}

In analogy to the package $S @ M$ [41] we have implemented three versions of the Schouten identity

$$
\langle i j\rangle\langle k l\rangle=\langle i l\rangle\langle k j\rangle+\langle i k\rangle\langle j l\rangle
$$

and its conjugated version.

\#Procedure Schouten $\left(p_{1}, p_{2}, q_{1}, q_{2}\right)$

Substitutes according to the Schouten identity

$$
\left\langle p_{1} p_{2}\right\rangle\left\langle q_{1} q_{2}\right\rangle \rightarrow\left\langle p_{1} q_{2}\right\rangle\left\langle q_{1} p_{2}\right\rangle+\left\langle p_{1} q_{1}\right\rangle\left\langle p_{2} q_{2}\right\rangle .
$$

\#Procedure Schouten $\left(p_{1}, p_{2}, q\right)$

Substitutes according to the Schouten identity

$$
\forall q^{\prime}:\left\langle p_{1} p_{2}\right\rangle\left\langle q q^{\prime}\right\rangle \rightarrow\left\langle p_{1} q^{\prime}\right\rangle\left\langle q p_{2}\right\rangle+\left\langle p_{1} q\right\rangle\left\langle p_{2} q^{\prime}\right\rangle .
$$

\section{\#Procedure Schouten $(q)$}

Substitutes according to the Schouten identity

$$
\forall p_{1}, p_{2}, p_{3}: \frac{\left\langle q p_{1}\right\rangle}{\left\langle q p_{2}\right\rangle\left\langle q p_{3}\right\rangle} \rightarrow \frac{\left\langle p_{2} p_{1}\right\rangle}{\left\langle q p_{2}\right\rangle\left\langle p_{2} p_{3}\right\rangle}-\frac{\left\langle p_{3} p_{1}\right\rangle}{\left\langle q p_{3}\right\rangle\left\langle p_{2} p_{3}\right\rangle} .
$$




\subsubsection{Miscellanous Routines}

In this section we describe some routines which are of less common use. The procedure SpClear nullifies all expressions which should vanish due to spinor lines of the wrong length. The procedure SpClear is called inside SpContract because Equations (15) hold only if the spinor chains have correct length.

\#Procedure SpClear()

Eliminates all terms which contain a spinor line $\langle p|\Gamma| q\rangle$ or

$[p|\Gamma| q]$, where $\Gamma$ is a product of an odd number of Dirac matrices, or a spinor line $\langle p|\Gamma| q]$ or $[p|\Gamma| q\rangle$, where $\Gamma$ is a product of an even number of Dirac matrices.

This procedure acts on expressions in collected form.

The routine SpCheck acts in a similar way as SpClear does. Instead of removing the terms which should vanish trivially it marks them with the function SpERRORTOKEN.

\#Procedure SpCheck ()

Substitutes all terms which contain a spinor line $\langle p|\Gamma| q\rangle$ or $[p|\Gamma| q]$, where $\Gamma$ is a product of an odd number of Dirac matrices, or a spinor line $\langle p|\Gamma| q]$ or $[p|\Gamma| q\rangle$, where $\Gamma$ is a product of an even number of Dirac matrices inside the arguments of the function SpERRORTOKEN.

This procedure acts on expressions in collected form.

The last procedure of this section, Sp0rder, shuffles Dirac matrices into a given order using the 't Hooft-Veltman algebra. The order is specified by the argument list of SpOrder. This routine is used in the test programs to bring the results into a canonical form which is necessary in order to check that all tests hold. 
\#Procedure SpOrder $\left(k_{1}, k_{2}, \ldots\right)$

Sorts all products of Dirac matrices according to the ordering specified by the argument list. This routine acts on non-commuting objects.

Parameters

$$
\begin{aligned}
& k_{1}, k_{2}, \ldots \text { a list of vectors. If } k_{i} \text { is to the left of } \\
& k_{j} \text { in the argument list then the procedure } \\
& \text { shuffles } k_{i} \text { to the left of } k_{j} \text { in the } \\
& \text { expression. }
\end{aligned}
$$

\subsection{Working with Majorana Spinors}

\subsubsection{On the Relative Sign of Feynman Graphs}

The flipping rules have been tested in Golem-2.0 where the diagrams are generated by Qgraf [47]. The relative sign calculated by Qgraf is incorrect when dealing with Majorana fermions. Here, we present a method to calculate it. Firstly we calculate $(-1)^{P}$ using the following code:

\section{Function NCOrder;}

Id fDUMMY1?\{UbarSpa, UbarSpb $\}$ (vDUMMY1?) =

NCOrder (vDUMMY1) *fDUMMY1 (vDUMMY1) ;

Id fDUMMY1?\{USpa, USpb $\}$ (vDUMMY1?) =

fDUMMY1 (vDUMMY1) * NCOrder (vDUMMY1) ;

\#call tHooftAlgebra

\#call SpCollect

ChainIn NCOrder;

AntiSymmetrize NCOrder;

Id $\operatorname{NCOrder}($ ? all $)=1$;

We multiply our diagram by a non-commuting function of the external momenta which encodes the order of the spinors in the diagram. The arguments of this function are then brought into Form's natural ordering. The exchange of any two arguments results in a minus sign.

Secondly we must determine $(-1)^{L}$ with $L$ being the number of closed fermion loops. This is easily done by counting the number of appearances of trL, as long as one ensures that the only source of spinor traces in the first place are closed fermion loops; the easiest way of multiplying the amplitude by the correct sign could be implemented as Id $\operatorname{trL}=-\operatorname{trL}$. 


\subsubsection{Fixing Fermion Chain Order}

The method we have described relies on being able to fix the fermion chain order. This is achieved in the procedure NCContainer. In our code an incoming (outgoing) Majorana fermion will initially be treated as in incoming (outgoing) Dirac fermion (as opposed to an anti-fermion). When we join an incoming Majorana spinor with an incoming Dirac fermion or an outgoing Dirac anti-fermion, one spinor will need to be flipped. The same applies when an outgoing Majorana fermion is joined to an incoming anti-fermion or an outgoing fermion. As an example we have an expression:

NCContainer (UbarSpa $(\mathrm{k} 1) * \operatorname{Sm}(\mathrm{i} 1) * \operatorname{Proj} \mathrm{Plus} * \operatorname{UbarSpa}(\mathrm{k} 2)$ )

which is transformed using RemoveNCContainer to

NCContainer (UbarSpa (k1), Sm(i1), ProjPlus, SpFlip (UbarSpa (k2)))

and then using (21) we have the fermion chain:

UbarSpa $(\mathrm{k} 1) * \operatorname{Sm}(\mathrm{i} 1) * \operatorname{ProjPlus} * \operatorname{USpb}(\mathrm{k} 2)$.

This is the default behaviour of spinney; one can prevent spinney from applying the flipping rules by defining the preprocessor variable NOSPFLIP before calling RemoveNCContainer.

\section{Examples and Applications}

\subsection{Feynman Diagram Based Reduction of One-Loop Amplitudes}

In this section we show how the Form library spinney can be used for the reduction of one-loop diagrams, both with conventional reduction of tensor integrals and with a reduction at the integrand level as described in [14]. In the first case we use the conventions of [48]. However, the method is not restricted to to this particular tensor decomposition.

In our setup, we use the diagram generator QGraf [47]. For the diagram in Figure 1 we obtain an output similar to the one given below 4

Local diagram $1=$
$\quad \operatorname{inp}(-1, \quad$ iv $1 \mathrm{r} 1 \mathrm{~L} 1, \mathrm{k} 1, \mathrm{me}) *$

\footnotetext{
${ }^{4}$ For the sake of simplicity we skip the discussion of the color algebra.
} 


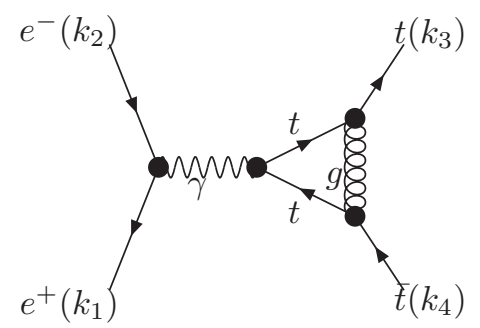

Figure 1: Three-point diagram $\left(e^{+} e^{-} \rightarrow t \bar{t}\right)$ discussed in the example.

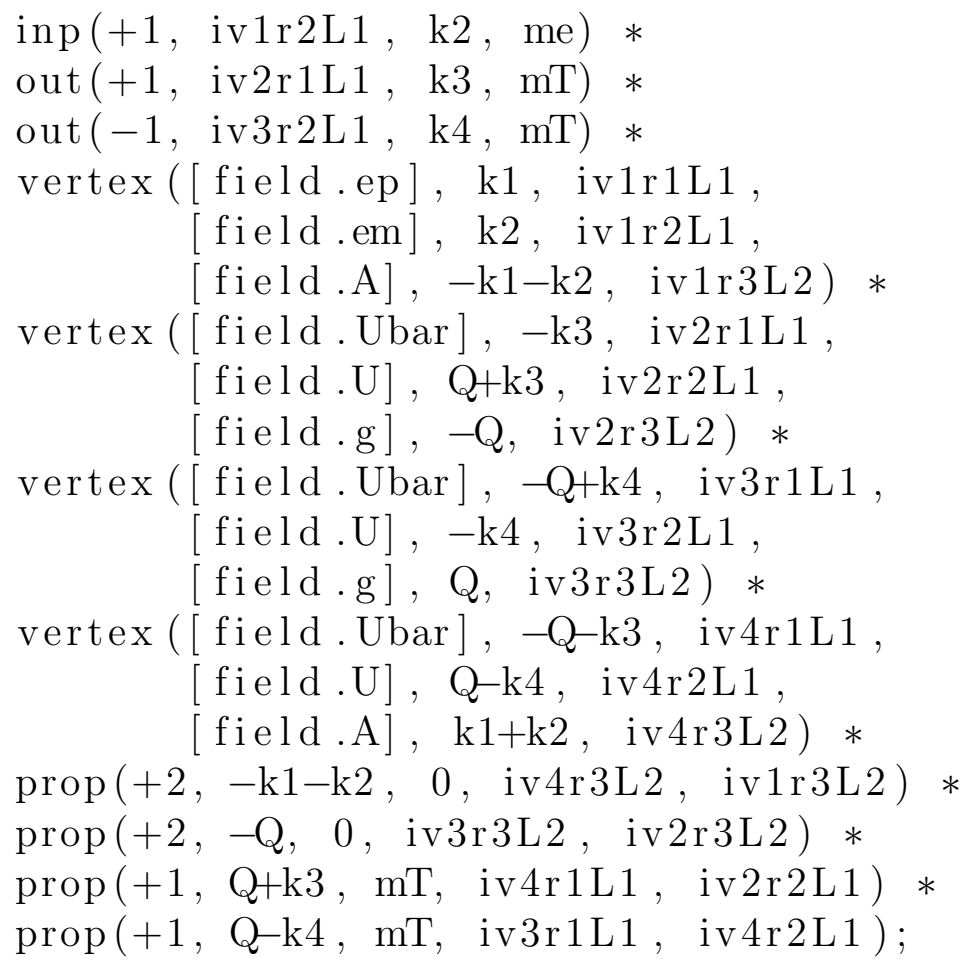

The first step is the substitution of the Feynman rules. In the replacement of the wave functions the approximation $m_{e}=0$ is applied and helicities are assigned to the particles.

Id $\operatorname{inp}(-1, \mathrm{iv} 1 ?, \mathrm{k} 1, \mathrm{me})=\mathrm{NCContainer}(\operatorname{UbarSpa}(\mathrm{k} 1)$, iv1);

Id $\operatorname{inp}(+1, \mathrm{iv} 1 ?, \mathrm{k} 2, \mathrm{me})=\mathrm{NCContainer}(\mathrm{USpb}(\mathrm{k} 2), \mathrm{iv} 1)$;

Id out $(+1, \mathrm{iv} 1 ?, \mathrm{k} 3, \mathrm{mT})=\mathrm{NCContainer}(\operatorname{UbarSpb}(\mathrm{k} 3,+1)$, iv 1$)$;

Id out $(-1, \mathrm{iv} 1 ?, \mathrm{k} 4, \mathrm{mT})=\mathrm{NCContainer}(\operatorname{USpa}(\mathrm{k} 4,-1)$, iv 1$)$; 
As discussed in Section 3.2.1 the letters ' $a$ ' and ' $b$ ' distinguish the helicities, whereas the parameter \pm 1 at the right-hand side differentiates between $u$ and $v$-spinors.

In a similar manner the propagators and vertices are substituted. Again, focussing on the Lorentz algebra we omit color structure, coupling constants and denominators here.

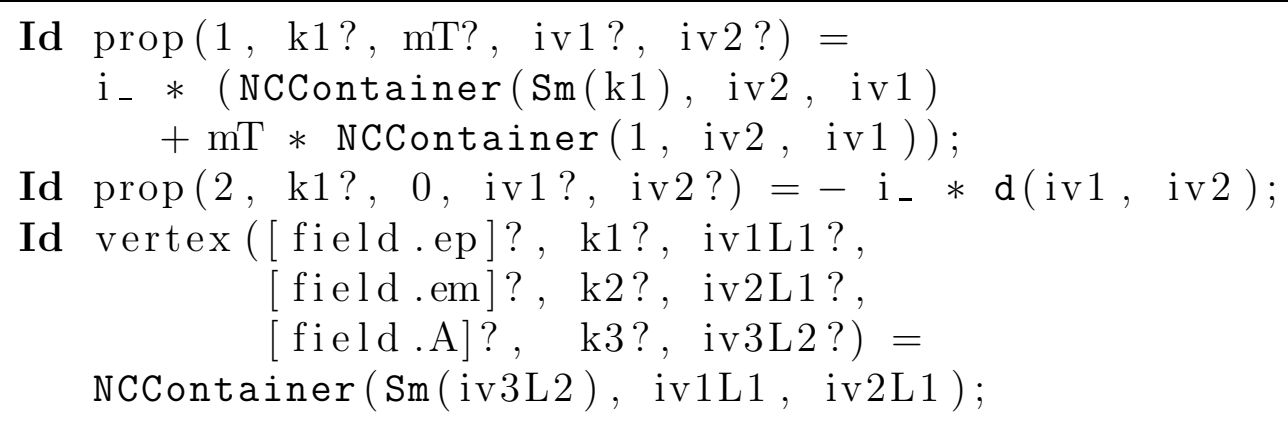

Now, a call to RemoveNCContainer brings the non-commuting object in the right order and simplifies the representation of the expression. We replace the massive spinors by projecting onto massless vectors. Hereby we introduce new vectors $l_{3}$ and $l_{4}$ as defined in Equations (7) and (8) .

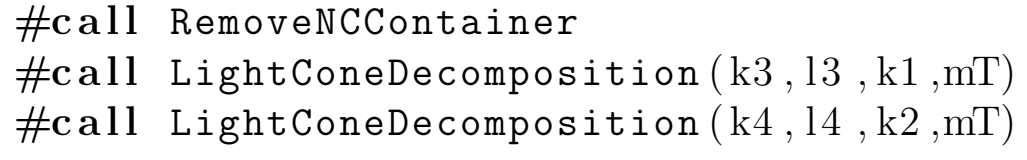

At this point the manipulation of the diagram branches depending on the output one wants to achieve. In a conventional reduction of the tensor integrals the algorithm contiues after replacing the integration momentum $Q$ by the corresponding expressions for the tensor integrals. In the case of a reduction at the integrand level we like to express $Q$ in terms of a fourdimensional projection $Q_{4}$ and a scale $\mu^{2}$ such that $Q^{2}=\left(Q_{4}\right)^{2}-\mu^{2}$.

\subsubsection{Conventional Tensor Reduction}

The expression corresponding to the Feynman diagram in Figure 1 has integrals of rank two at most. Following the notation of [48] the momenta in the loop are $r_{1}=-k_{4}, r_{2}=k_{3}$ and $r_{3}=0$. The corresponding form factor 
representation of the occurring tensor integrals is

$$
\begin{aligned}
I_{3}^{n}(S) & =A^{3,0}(S) \\
I_{3}^{n, \mu_{1}}(S) & =-k_{4}^{\mu_{1}} A_{1}^{3,1}(S)+k_{3}^{\mu_{1}} A_{2}^{3,1}(S) ; \\
I_{3}^{n, \mu_{1} \mu_{2}}(S) & =k_{4}^{\mu_{1}} k_{4}^{\mu_{2}} A_{11}^{3,2}(S)-\left(k_{3}^{\mu_{1}} k_{4}^{\mu_{2}}+k_{4}^{\mu_{1}} k_{3}^{\mu_{2}}\right) A_{12}^{3,2}(S) \\
& +k_{4}^{\mu_{1}} k_{4}^{\mu_{2}} A_{22}^{3,2}(S)+g^{\mu_{1} \mu_{2}} B^{3,2}(S) .
\end{aligned}
$$

These formulae are easily implemented for the given example. It should be noted that the calls to the function LightConeDecomposition must be repeated after this step since we reintroduce the momenta $k_{3}$ and $k_{4}$ here.

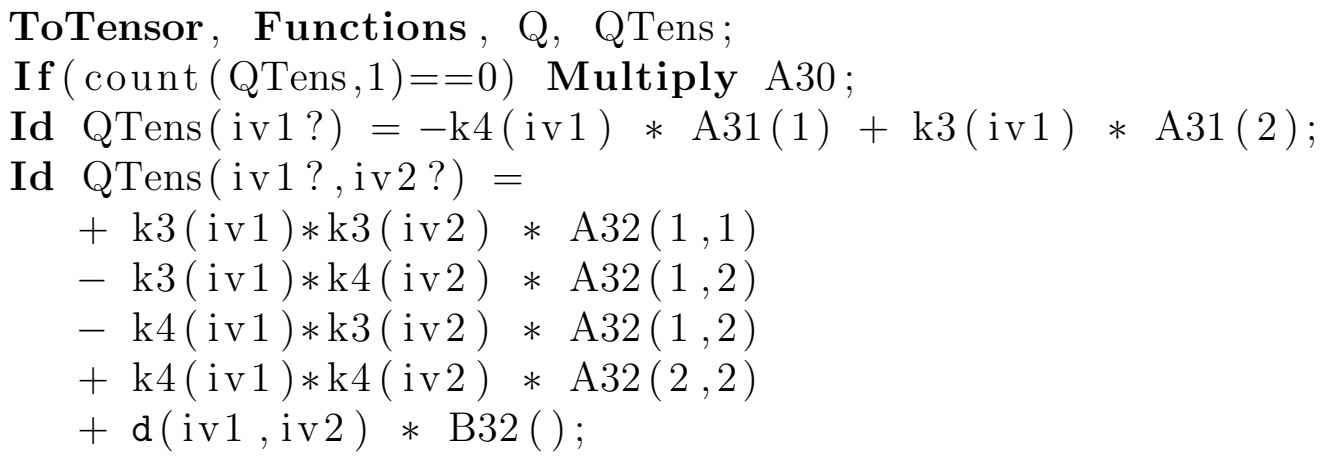

The remaining steps of the program carry out the 't Hooft algebra and simplify the expression by taking out all contractions of Lorentz indices. The replacements of $\mathrm{dEps}$ are consistent with the dimension splitting in use and define the symbol eps such that $n=4-2 \varepsilon$. In the four-dimensional helicity (FDH) scheme [49 53] the symbol eps can be replaced by zero; in the 't Hooft-Veltman scheme the products between $\varepsilon$ (resp. $\varepsilon^{2}$ ) and the form factors, which are formally Laurent series in $\varepsilon$, lead to rational terms. After these steps the diagram is in a form suitable for numerical evaluation if one provides routines for the computation of spinor brackets $(\langle\cdot \cdot\rangle$ and $[\cdot \cdot])$ and a library of integral form factors such as golem95 [54].

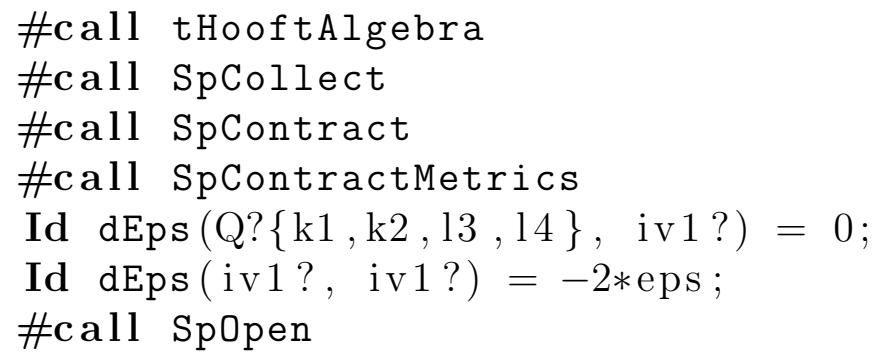




\subsubsection{Reduction at the Integrand Level}

Following the strategy of reducing the Feynman diagrams at the integrand level, we show in this section how the numerator of the Feynman diagram can be constructed very easily by the use of spinney. We consider two different approaches corresponding to two publicly available reduction packages. The strategy implemented in CutTools [55] uses a numerator function $N\left(Q_{4}\right)$ which only depends on the four-dimensional projection of the (complex) integration momentum $Q$. This method allows for the reconstruction of the cut-constructible terms but only partially recovers the rational parts of an amplitude. This is due to the fact that terms in $\mu^{2}$, where $Q^{2}=Q_{4}^{2}-\mu^{2}$, lead to rational terms which are not taken into account and need to be added by a separate calculation [56, 57]. An improved reduction at the integrand level has been implemented by the authors of Samurai [15]. This method takes advantage of a numerator function depending on both $Q_{4}$ and $\mu^{2}$, which can be decomposed into

$$
N\left(Q_{4}, \mu^{2}\right)=N_{0}\left(Q_{4}, \mu^{2}\right)+\varepsilon N_{1}\left(Q_{4}, \mu^{2}\right)+\varepsilon^{2} N_{2}\left(Q_{4}, \mu^{2}\right)+\mathcal{O}\left(\varepsilon^{3}\right) .
$$

As in the case of the conventional tensor reduction, $\varepsilon$ is set to zero (keeping terms in $\mu^{2}$ ) in the FDH scheme, whereas in the 't-Hooft Veltman scheme the independent reduction of the numerators $N_{0}, N_{1}$ and $N_{2}$, multiplication with the apropriate terms of the Laurent series of the scalar integrals leads to the full result, including both both the cut-constructible and the rational part of the amplitude.

Irrespective of the approach chosen the first step to be taken should be to carry out the 't Hooft algebra. Then the two methods differ in the way they deal with terms in dEps. Using CutTools these terms are neglected and set to zero. In the case of Samurai one substitutes the relations which are implied by $Q^{2}=Q_{4}^{2}-\mu^{2}$, where $g^{\mu \nu} Q^{2}=Q_{\mu} Q_{\nu}$ and $Q_{4}^{\mu}=\hat{g}^{\mu \nu} Q_{\nu}$, and therefore $\mu^{2}=-\tilde{g}^{\mu \nu} Q_{\mu} Q_{\nu}$.

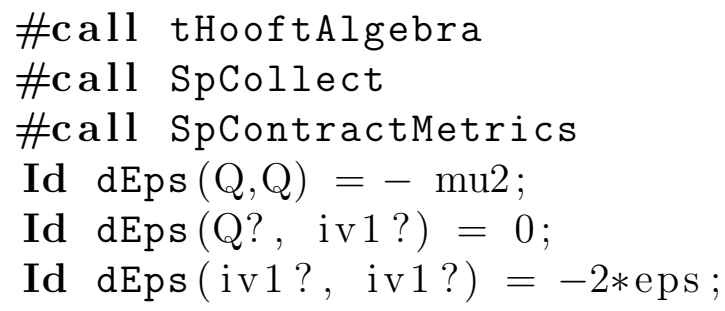




$$
\begin{aligned}
& \text { EndArgument; } \\
& \text { Id } \mathrm{Q} \cdot \mathrm{Q}=\mathrm{Q} 4 . \mathrm{Q} 4-\mathrm{mu} 2 \\
& \text { Id } \mathrm{Q}=\mathrm{Q} 4 ; \\
& \text { Id } \mathrm{d} 4(\mathrm{k} 1 \text { ? }, \mathrm{i} \mathrm{v} 1 ?)=\mathrm{k} 1(\mathrm{iv} 1)
\end{aligned}
$$

Finally, one can contract the remaining Lorentz indices and bring the the expression into a form where spinor brackets $\left(\langle\cdot \cdot\rangle,[\cdot \cdot]\right.$ and $\left.\left[\cdot\left|\phi_{4}\right| \cdot\right\rangle\right)$ are the only functions to be evaluated numerically.

\#call spContract

\#call Sp0pen $(\mathrm{k} 1, \mathrm{k} 2,13,14)$

\subsection{Working with Majorana Spinors}

\subsubsection{Charge Conjugation of a Vector Current}

We illustrate our approach through the following simple example. The vector current, $\left\langle p^{+}\left|\gamma^{\mu}\right| q^{+}\right\rangle$, satisfies the charge conjugation relation

$$
\left\langle p^{+}\left|\gamma^{\mu}\right| q^{+}\right\rangle=\left\langle q^{-}\left|\gamma^{\mu}\right| p^{-}\right\rangle
$$

which we can show explicitly using charge conjugation relations. This charge conjugation operation is equivalent to us reversing the fermion flow arrow. Therefore we can use Equations (20) to show this equivalence holds:

$$
\begin{aligned}
\left\langle p^{+}\left|\gamma^{\mu}\right| q^{+}\right\rangle & =[p|\mu| q\rangle \rightarrow(-1)^{P}\left\langle q\left|\gamma^{\mu^{\prime}}\right| p\right]=(-1)^{P}(-1)\left\langle q\left|\gamma^{\mu}\right| p\right] \\
& =\left\langle q\left|\gamma^{\mu}\right| p\right]=\left\langle q^{-}\left|\gamma^{\mu}\right| p^{-}\right\rangle
\end{aligned}
$$

where we have used (19) and (21) and we have defined our reference order as $(p, q)$ giving $(-1)^{P}=-1$. This result is true for both Dirac and Majorana fermions.

\subsubsection{Majorana Exchange}

We now consider two Dirac fermions scattering to two vector bosons via a t-channel Majorana fermion exchange. There are two equivalent orientations to choose from as shown in Figure 2, We write our amplitude as

$$
\mathcal{A}=\mathcal{A}^{\mu \nu} \epsilon_{\mu}^{*}\left(p_{3}\right) \epsilon_{\mu}^{*}\left(p_{4}\right)
$$

Our first choice of orientation gives

$$
\mathcal{A}_{1}^{\mu \nu}=\left\langle p_{1}\left|\left(\gamma^{\mu}\right)^{\prime} S(p) \gamma^{\nu}\right| p_{2}\right\rangle
$$



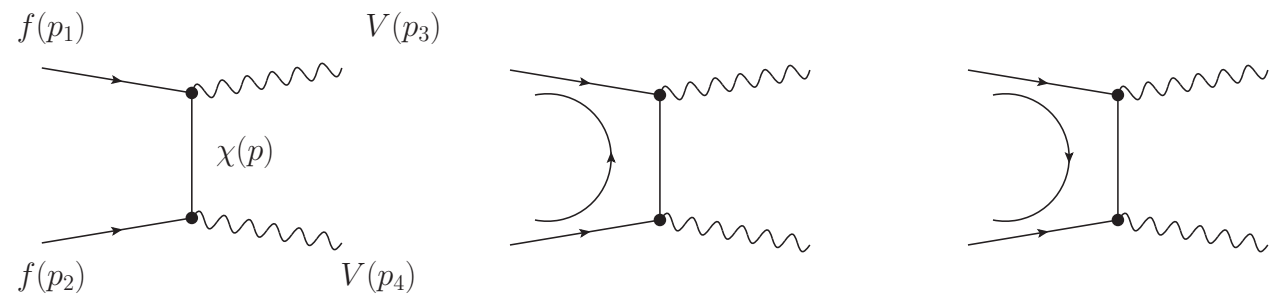

Figure 2: The process $(f f \rightarrow V V)$ mediated by the exchange of a Majorana fermion $(\chi)$ as discussed in the example. The left hand figure shows the original diagram; the middle figure shows the first choice of fermion orientation; and the figure on the right shows the second choice of orientation.

and the second orientation gives

$$
\mathcal{A}_{2}^{\mu \nu}=(-1)\left\langle p_{2}\left|\left(\gamma^{\nu}\right)^{\prime} S(-p) \gamma^{\mu}\right| p_{1}\right\rangle
$$

where we have chosen the reference order $\left(p_{1}, p_{2}\right)$. Applying our flipping rules to Equation (37) gives the result:

$$
\mathcal{A}_{1}^{\mu \nu^{\prime}}=(-1)^{P}\left\langle p_{2}\left|\left(\gamma^{\nu}\right)^{\prime} S(-p) \gamma^{\mu}\right| p_{1}\right\rangle=\mathcal{A}_{2}^{\mu \nu} .
$$

Therefore the amplitude is independent of the original choice of fermion orientation.

In our code, part of the output is:

NCContainer (USpa $(\mathrm{k} 1) * \operatorname{SpFlip}(\mathrm{Sm}(\mathrm{mu}) *$ ProjPlus $) * \operatorname{Sm}(\mathrm{k} 4)$

$* \operatorname{Sm}(\mathrm{nu}) * \operatorname{ProjPlus} * \operatorname{USpb}(\mathrm{k} 2)) * \operatorname{inv}($ es 23$)$.

Upon applying the RemoveNCContainer routine we obtain the result

- UbarSpb $(\mathrm{k} 1) * \operatorname{ProjPlus} * \operatorname{Sm}(\mathrm{mu}) * \operatorname{Sm}(\mathrm{k} 4) * \operatorname{Sm}(\mathrm{nu})$

*ProjMinus *USpa $(\mathrm{k} 2) * \operatorname{inv}($ es 23$)$

We have picked up a minus sign from the flipping of the $\gamma^{\mu}$. What remains is to multiply by $(-1)^{P}$ as explained previously.

\subsection{Coefficients of Scalar Integrals by Unitarity Based Methods}

In this example we consider Bhaba scattering in QED at the one-loop level. We use the well known fact that any (leg-ordered) one-loop amplitude 
can be written in terms of a basis of scalar one-loop integrals,

$$
\begin{array}{r}
\mathcal{A}_{\text {1-loop }}=\sum_{i_{1}} a_{i_{1}} \int \frac{\mathrm{d}^{n} l}{D_{i_{1}}}+\sum_{i_{1}<i_{2}} b_{i_{1} i_{2}} \int \frac{\mathrm{d}^{n} l}{D_{i_{1}} D_{i_{2}}}+\sum_{i_{1}<i_{2}<i_{3}} c_{i_{1} i_{2} i_{3}} \int \frac{\mathrm{d}^{n} l}{D_{i_{1}} D_{i_{2}} D_{i_{3}}} \\
+\sum_{i_{1}<i_{2}<i_{3}<i_{4}} d_{i_{1} i_{2} i_{3} i_{4}} \int \frac{\mathrm{d}^{n} l}{D_{i_{1}} D_{i_{2}} D_{i_{3}} D_{i_{4}}},
\end{array}
$$

where $D_{i}=\left[\left(l+r_{i}\right)^{2}-m_{i}^{2}\right]$.

We consider the two diagrams in Fig. 3. The box coefficient is isolated by performing four cuts, which completely disconnect the loop amplitude into 4 tree-level partial amplitudes with three external legs each. In fact, since there is only one vertex in QED and the identity of one of the legs is fixed (it is one of the original external fermions), we have two possible contributions in each amplitude differing by the exchange of the remaining fermion/photon legs of the QED vertex. Consistency, i.e. the fact that the lines connecting neighbouring vertices need obviously be of the same type, reduces this to two choices, depicted on the left-hand side of Fig. 3. The helicity conservation on the fermion lines and the restrictions of complex kinematics allow us to write down the only four possible internal helicity configurations. The four cuts also provide four independent constraints on the loop momentum, thus determining all its components (in fact there are two solutions). For details see [22]. We take all momenta to be outgoing.

We begin the computation by preparing a small procedure that will be used repeatedly together with SpOpen to simplify denominator structures. It is an ad-hoc solution based on the knowledge of type and depth of denominators arising due to loop momenta substitutions.

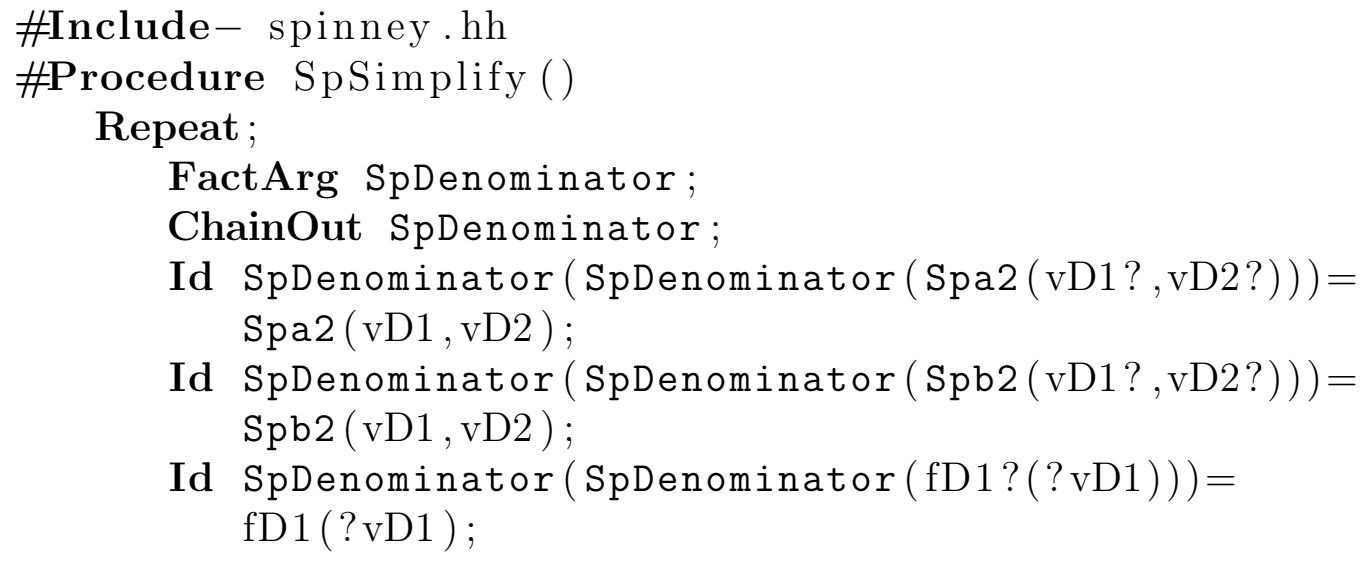



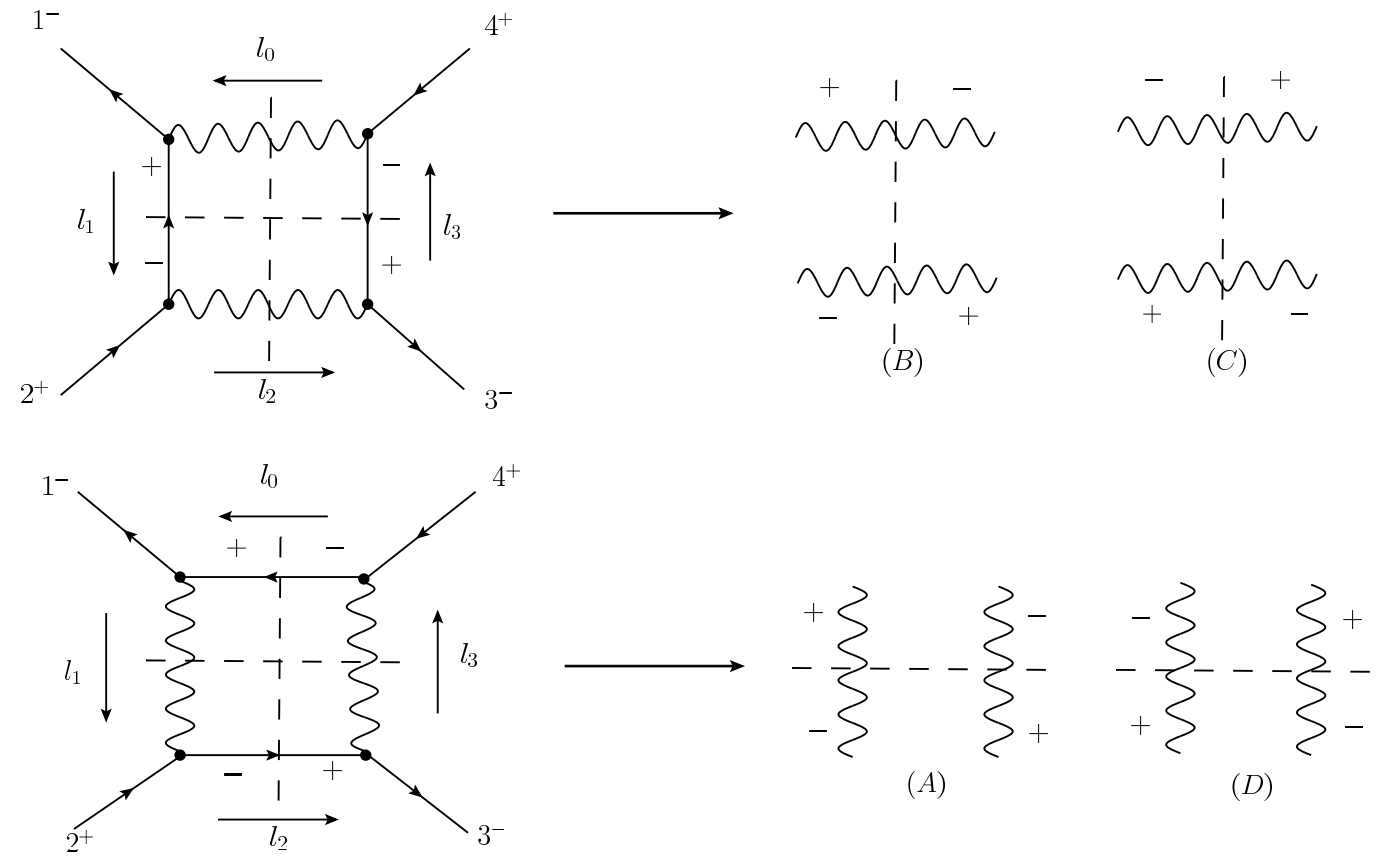

(A)

$(D)$

Figure 3: Possible helicity configurations of the two box diagrams contributing to the $f \bar{f} \rightarrow f \bar{f}$ amplitude.

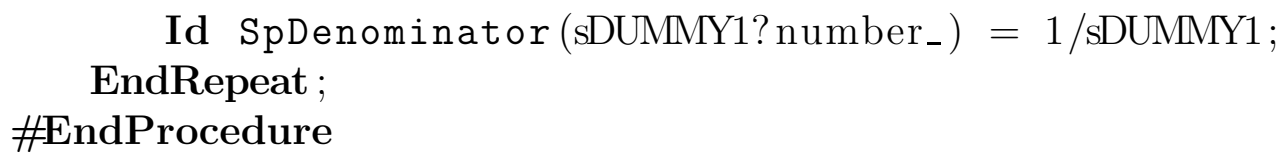

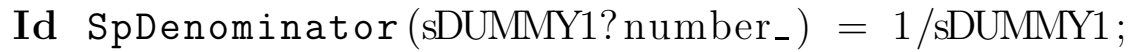
EndRepeat ; \#EndProcedure

We write down the explicit formula for one of the four internal helicity configurations, split into separate numerator and denominator expressions. The formulae for the remaining configurations are similar.

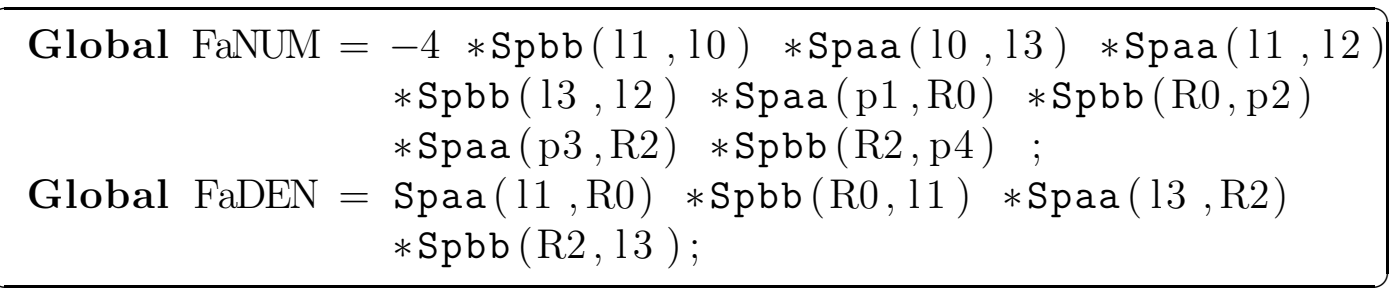

The first step is to substitute the solutions for the loop momentum on the cut, which in our case is:

$$
l_{0}^{\mu}=\frac{1}{2} \frac{[p 2 p 1]}{[p 2 p 4]}\left\langle p 1\left|\gamma^{\mu}\right| p 4\right]
$$


In order to do so we need to re-express all loop momenta in terms of $l_{0}$ and external momenta. We use SpClose to have the spinor products in a form which is suitable for standard linear substitutions: $\left\langle p\left|\ldots l_{i_{1}} \ldots l_{i_{2}} \ldots\right| q\right\rangle$, with $p$ and $q$ being external momenta and $l_{i}$ the loop momentum. This might not be immediately possible, as in our example, where SpClose produces an output of the type $\left\langle l_{i_{1}}|\ldots| l_{i_{2}}\right\rangle$ instead. However, a call to Schouten solves the problem at the cost of having more terms in the calculation.

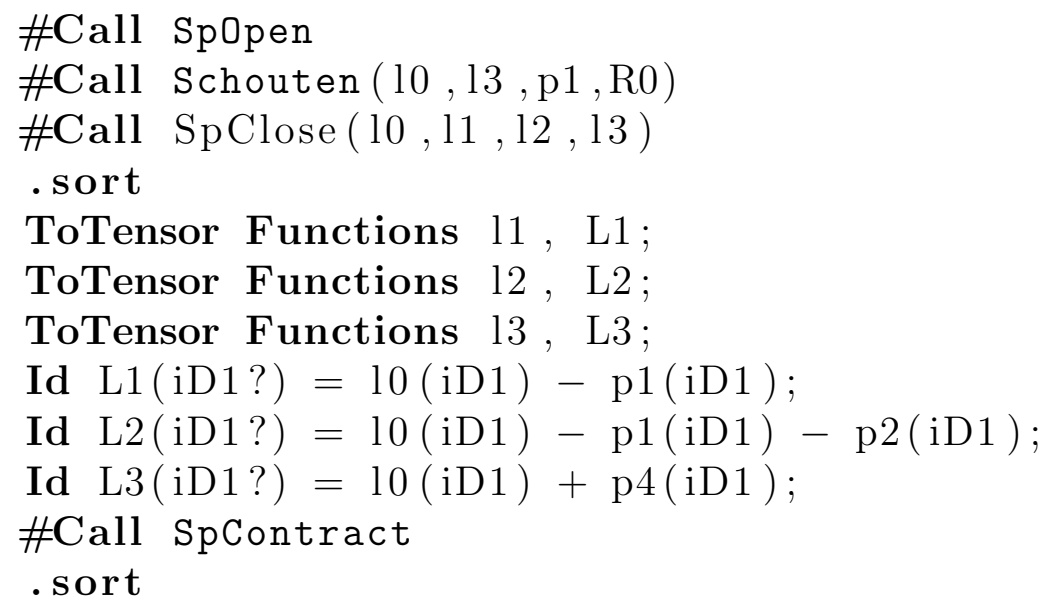

Now we substitute the solution for $l_{0}^{\mu}$ and contract all explicit Lorentz indices.

ToTensor Functions 10, L0;

ChainOut L0;

. sort

Id $\mathrm{L} 0(\mathrm{iD} 1 ?)=\operatorname{Spab}(\mathrm{p} 1, \mathrm{iD} 1, \mathrm{p} 4) * \operatorname{Spb} 2(\mathrm{p} 2, \mathrm{p} 1) *(1 / 2) *$ SpDenominator ( Spb2 (p2, p4));

\#Call SpContractMetrics

\#Call spcontract

\#Call SpOpen

. sort

We combine the numerator and denominator expressions obtained before. Our formulae still involve the reference momenta of the photons, which is our gauge freedom - we can greatly simplify the computations by making an explicit choice at this point.

Global $\mathrm{Fa}=\mathrm{FaNUM} * \mathrm{SpDenominat}$ or $(\mathrm{FaDEN})$; Argument; 


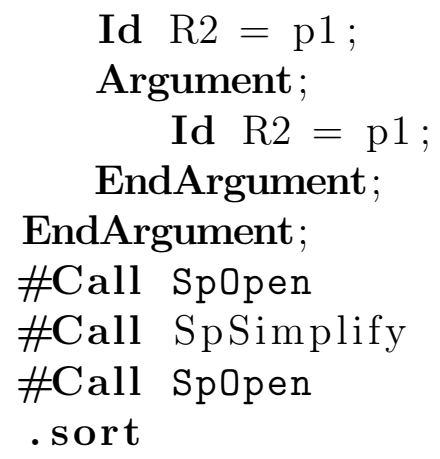

As a finishing touch, we apply the following sequence of transformations to get rid of any remaining double denominator-type expressions, which appear upon introducing the explicit form of the $l_{0}$ loop momentum.

\section{Argument;}

\#Call Schouten (p1,p2,p4,R0)

\section{EndArgument;}

\section{Repeat;}

$$
\text { \#Call spopen () }
$$

Argument;

$$
\text { \#Call SpOpen () }
$$

\section{EndArgument;}

\#Call SpSimplify

\section{EndRepeat ;}

\section{. store}

Finally, we write down the expression for the complete box coefficient. Note the factor $1 / 2$, which comes from averaging over two solutions to the loop momentum constraints.

Local $\mathrm{F}=(1 / 2) *(\mathrm{Fa}+\mathrm{Fb}+\mathrm{Fc}+\mathrm{Fd})$;

Print $\mathrm{F}$;

.end

The FORM output we obtain is:

$$
\begin{aligned}
F=-\frac{2\left\langle p_{1} p_{2}\right\rangle^{3}\left\langle p_{3} p_{4}\right\rangle\left[p_{2} p_{1}\right]^{2}}{\left\langle p_{2} p_{4}\right\rangle^{2}}+\frac{2\left\langle p_{1} p_{4}\right\rangle^{3}\left\langle p_{2} p_{3}\right\rangle\left[p_{4} p_{1}\right]^{2}}{\left\langle p_{2} p_{4}\right\rangle^{2}} \\
+2\left\langle p_{1} p_{2}\right\rangle\left\langle p_{1} p_{3}\right\rangle\left[p_{2} p_{1}\right]\left[p_{4} p_{2}\right]-2\left\langle p_{1} p_{3}\right\rangle\left\langle p_{1} p_{4}\right\rangle\left[p_{4} p_{1}\right]\left[p_{4} p_{2}\right] .
\end{aligned}
$$




\section{Conclusion}

In this article we have presented a new Form library for processing expressions containing helicity spinors in four and $n=(4-2 \varepsilon)$ dimensions. For the $n$-dimensional algebra we have implemented the 't-Hooft algebra with dimension splitting. This gives full flexibility to the user about the choice of the regularisation scheme, as in many schemes different from the 't HooftVeltman scheme it is sufficient to neglect terms in $\varepsilon$ which stem from the numerator algebra.

In various examples we have shown that the new package is applicable to calculations both using traditional and modern, unitarity based methods. The provided routines implement the typical steps which are necessary for an algebraic simplification of helicity amplitudes and therefore simplify the task of implementing such calculations. The implementation of flipping rules for Majorana spinors allows to extend the domain of applicability to theories beyond the Standard Model.

\section{Acknowledgements}

T.R. wants to thank Ralf Sattler for useful discussion. T.R. is supported by the Dutch Foundation for Fundamental Research on Matter (FOM), project FORM 07PR2556. GC would like to thank Nikhef for their hospitality while part of this work was carried out. G.C. is supported by the British Science and Technology Facilities Council (STFC).

\section{References}

[1] Xu, Z., Zhang, D.-H., and Chang, L., Nucl. Phys. B291 (1987) 392.

[2] Berger, C. F. et al., Nucl. Phys. Proc. Suppl. 183 (2008) 313, [0807.3705].

[3] Berger, C. F. et al., Phys. Rev. Lett. 102 (2009) 222001, [0902.2760].

[4] Berger, C. F. et al., Phys. Rev. D80 (2009) 074036, [0907.1984].

[5] Berger, C. F. et al., (2010), [1004.1659].

[6] Giele, W. T. and Zanderighi, G., JHEP 06 (2008) 038, [0805.2152]. 
[7] Ellis, R. K., Giele, W. T., Kunszt, Z., and Melnikov, K., Nucl. Phys. B822 (2009) 270, [0806.3467].

[8] Ellis, R. K., Melnikov, K., and Zanderighi, G., JHEP 04 (2009) 077, [0901.4101].

[9] Keith Ellis, R., Melnikov, K., and Zanderighi, G., Phys. Rev. D80 (2009) 094002, [0906.1445].

[10] Bevilacqua, G., Czakon, M., Papadopoulos, C. G., Pittau, R., and Worek, M., JHEP 09 (2009) 109, [0907.4723].

[11] Bevilacqua, G., Czakon, M., Papadopoulos, C. G., and Worek, M., Phys. Rev. Lett. 104 (2010) 162002, [1002.4009].

[12] van Hameren, A., Papadopoulos, C. G., and Pittau, R., JHEP 09 (2009) 106, [0903.4665].

[13] van Hameren, A., JHEP 07 (2009) 088, [0905.1005].

[14] Ossola, G., Papadopoulos, C. G., and Pittau, R., Nucl. Phys. B763 (2007) 147, hep-ph/0609007.

[15] Mastrolia, P., Ossola, G., Reiter, T., and Tramontano, F., (2010), [1006.0710].

[16] Melia, T., Melnikov, K., Rontsch, R., and Zanderighi, G., (2010), [1007.5313].

[17] Denner, A. and Dittmaier, S., Nucl. Phys. B734 (2006) 62, hep-ph/0509141.

[18] Denner, A., Dittmaier, S., Roth, M., and Wieders, L. H., Nucl. Phys. B724 (2005) 247, hep-ph/0505042.

[19] Bredenstein, A., Denner, A., Dittmaier, S., and Pozzorini, S., Phys. Rev. Lett. 103 (2009) 012002, 0905.0110].

[20] Bredenstein, A., Denner, A., Dittmaier, S., and Pozzorini, S., JHEP 03 (2010) 021, [1001.4006].

[21] Binoth, T. et al., Phys. Lett. B685 (2010) 293, 0910.4379]. 
[22] Britto, R., Cachazo, F., and Feng, B., Nucl. Phys. B725 (2005) 275, hep-th/0412103.

[23] Britto, R., Feng, B., and Mastrolia, P., Phys. Rev. D73 (2006) 105004, hep-ph/0602178.

[24] Mastrolia, P., Phys. Lett. B644 (2007) 272, hep-th/0611091.

[25] Forde, D., Phys. Rev. D75 (2007) 125019, [0704.1835].

[26] Bjerrum-Bohr, N. E. J., Dunbar, D. C., and Perkins, W. B., JHEP 04 (2008) 038, [0709.2086].

[27] Kilgore, W. B., (2007), [0711.5015].

[28] Badger, S. D., JHEP 01 (2009) 049, [0806.4600].

[29] Mastrolia, P., Phys. Lett. B678 (2009) 246, [0905.2909].

[30] Mastrolia, P., Lett. Math. Phys. 91 (2010) 199, [0906.3789].

[31] Berger, C. F., Del Duca, V., and Dixon, L. J., Phys. Rev. D74 (2006) 094021, hep-ph/0608180.

[32] Badger, S. D. and Glover, E. W. N., Nucl. Phys. Proc. Suppl. 160 (2006) 71, hep-ph/0607139.

[33] Badger, S. D., Glover, E. W. N., and Risager, K., JHEP 07 (2007) 066, [0704.3914].

[34] Glover, E. W. N., Mastrolia, P., and Williams, C., JHEP 08 (2008) 017, [0804.4149].

[35] Badger, S., Nigel Glover, E. W., Mastrolia, P., and Williams, C., JHEP 01 (2010) 036, [0909.4475].

[36] Dixon, L. J. and Sofianatos, Y., JHEP 08 (2009) 058, [0906.0008].

[37] Badger, S., Campbell, J. M., Ellis, R. K., and Williams, C., JHEP 12 (2009) 035, [0910.4481].

[38] Andersen, J. R. et al., (2010), [1003.1241]. 
[39] Bern, Z. et al., (2008), [0803.0494].

[40] Vermaseren, J. A. M., (2000), math-ph/0010025.

[41] Maitre, D. and Mastrolia, P., Comput. Phys. Commun. 179 (2008) 501, [0710.5559].

[42] Reiter, T., (2009), [0903.0947], PhD Thesis, The University of Edinburgh.

[43] Haber, H. E. and Kane, G. L., Phys. Rept. 117 (1985) 75.

[44] Denner, A., Eck, H., Hahn, O., and Kublbeck, J., Nucl. Phys. B387 (1992) 467.

[45] Hahn, T., Comput. Phys. Commun. 140 (2001) 418, hep-ph/0012260].

[46] Briggs, P., Ramsdell, J. D., and Mengel, M. W., Nuweb Version 1.0b1: A Simple Literate Programming Tool.

[47] Nogueira, P., J. Comput. Phys. 105 (1993) 279.

[48] Binoth, T., Guillet, J. P., Heinrich, G., Pilon, E., and Schubert, C., JHEP 10 (2005) 015, hep-ph/0504267.

[49] Bern, Z. and Kosower, D. A., Nucl. Phys. B379 (1992) 451.

[50] Kunszt, Z., Signer, A., and Trocsanyi, Z., Nucl. Phys. B411 (1994) 397, hep-ph/9305239.

[51] Catani, S., Seymour, M. H., and Trocsanyi, Z., Phys. Rev. D55 (1997) 6819, hep-ph/9610553.

[52] Catani, S., Dittmaier, S., and Trocsanyi, Z., Phys. Lett. B500 (2001) 149, hep-ph/0011222.

[53] Signer, A. and Stockinger, D., Nucl. Phys. B808 (2009) 88, [0807.4424].

[54] Binoth, T., Guillet, J. P., Heinrich, G., Pilon, E., and Reiter, T., Comput. Phys. Commun. 180 (2009) 2317, [0810.0992].

[55] Ossola, G., Papadopoulos, C. G., and Pittau, R., JHEP 03 (2008) 042, [0711.3596]. 
[56] Draggiotis, P., Garzelli, M. V., Papadopoulos, C. G., and Pittau, R., JHEP 04 (2009) 072, [0903.0356].

[57] Garzelli, M. V., Malamos, I., and Pittau, R., JHEP 01 (2010) 040, [0910.3130]. 